\title{
The Aurora volcanic field, California-Nevada: oxygen fugacity constraints on the development of andesitic magma
}

Received: 7 July 1995 / Accepted: 19 April 1996

\begin{abstract}
The Aurora volcanic field, located along the northeastern margin of Mono Lake in the Western Great Basin, has erupted a diverse suite of high-K and shoshonitic lava types, with 48 to $76 \mathrm{wt} \% \mathrm{SiO}_{2}$, over the last 3.6 million years. There is no correlation between the age and composition of the lavas. Three-quarters of the volcanic field consists of evolved $(<4 \mathrm{wt} \% \mathrm{MgO})$ basaltic andesite and andesite lava cones and flows, the majority of which contain sparse, euhedral phenocrysts that are normally zoned; there is no evidence of mixed, hybrid magmas. The average eruption rate over this time period was $\sim 200 \mathrm{~m}^{3} / \mathrm{km}^{2} /$ year, which is typical of continental arcs and an order of magnitude lower than that for the slow-spreading mid-Atlantic ridge. All of the Aurora lavas display a trace-element signature common to subduction-related magmas, as exemplified by $\mathrm{Ba} / \mathrm{Nb}$ ratios between 52 and 151 . Pre-eruptive water contents ranged from $1.5 \mathrm{wt} \%$ in plagioclase-rich two-pyroxene andesites to $\sim 6 \mathrm{wt} \%$ in a single hornblende lamprophyre and several biotite-hornblende andesites. Calculated oxygen fugacities fall within -0.4 and $+2.4 \log$ units of the Ni-NiO buffer. The Aurora potassic suite follows a classic, calc-alkaline trend in a plot of $\mathrm{FeO}^{\mathrm{T}} / \mathrm{MgO}$ vs $\mathrm{SiO}_{2}$ and displays linear decreasing trends in $\mathrm{FeO}^{\mathrm{T}}$ and $\mathrm{TiO}_{2}$ with $\mathrm{SiO}_{2}$ content, suggesting a prominent role for $\mathrm{Fe}-\mathrm{Ti}$ oxides during differentiation. However, development of the calc-alkaline trend through fractional crystallization of titanomagnetite would have caused the residual liquid to become so depleted in ferric iron that its oxygen fugacity would have fallen several log units below that of the Ni-NiO buffer. Nor can fractionation of hornblende be invoked, since it has the same effect as titanomagnetite in depleting the residual liquid in ferric
\end{abstract}

R.A. Lange $(\varpi)$

Department of Geological Sciences, University of Michigan, Ann Arbor, MI 48109, USA

I.S.E. Carmichael

Department of Geology and Geophysics,

University of California, Berkeley, CA 94720, USA

Editorial responsibility: T.L. Grove iron, together with a thermal stability limit that is lower than the eruption temperatures of several andesites ( $\sim 1040-1080^{\circ} \mathrm{C}$; derived from two-pyroxene thermometry). Unless some progressive oxidation process occurs, fractionation of titanomagnetite or hornblende cannot explain a calc-alkaline trend in which all erupted lavas have oxygen fugacites $\geq$ the $\mathrm{Ni}-\mathrm{NiO}$ buffer. In contrast to fractional crystallization, closed-system equilibrium crystallization will produce residual liquids with an oxygen fugacity that is similar to that of the initial melt. However, the eruption of nearly aphryic lavas argues against tapping from a magma chamber during equilibrium crystallization, a process that requires crystals to remain in contact with the liquid. A preferred model involves the accumulation of basaltic magmas at the mantle-crust interface, which solidify and are later remelted during repeated intrusion of basalt. As an endmember case, closed-system equilibrium crystallization of a basalt, followed by equilibrium partial melting of the gabbro will produce a calc-alkaline evolved liquid (namely, high $\mathrm{SiO}_{2}$ and low $\mathrm{FeO}^{\mathrm{T}} / \mathrm{MgO}$ ) with a relative $f_{\mathrm{O}_{2}}$ (corrected for the effect of changing temperature) that is similar to that of the initial basalt. Differentiation of the Aurora magmas by repeated partial melting of previous underplates in the lower crust rather than by crystal fractionation in large, stable magma chambers is consistent with the low eruption rate at the Aurora volcanic field.

\section{Introduction}

The Aurora volcanic field $\left(\sim 325 \mathrm{~km}^{2}\right)$ is located along the northern margin of the Mono Basin, adjacent to the eastern escarpment of the central Sierra Nevada and straddles the Nevada-California boundary near the historic gold mining towns of Bodie and Aurora (Fig. 1). It resides within the Basin and Range extensional province of the western United States, which has long been considered a classic locality for bimodal (basalt-rhyolite) volcanism. Thus, the area around the Mono Basin is well 


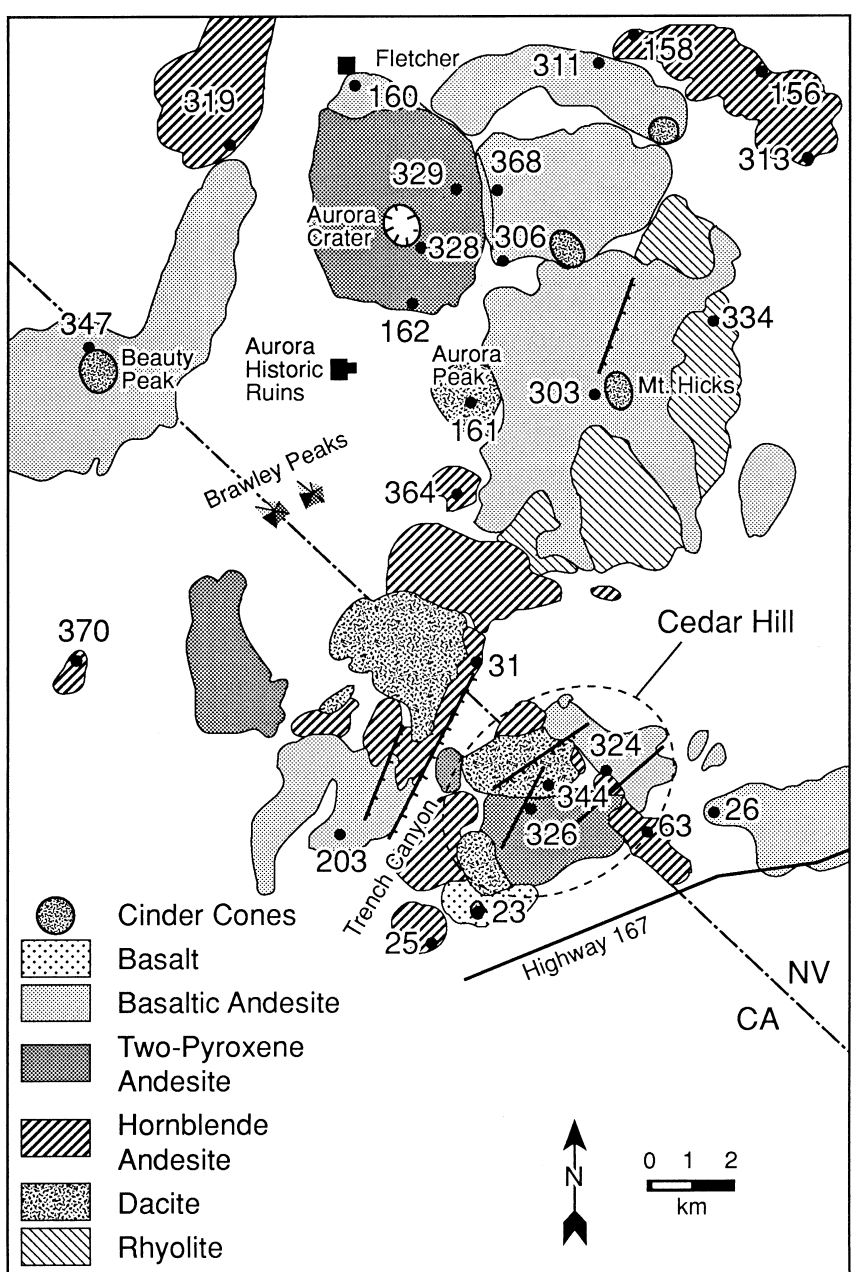

Fig. 1 Geologic map of the Aurora volcanic field; mapped by the authors. Samples discussed in the text are labelled known for its Quaternary rhyolitic volcanism (e.g., Mono Craters, Inyo domes, Bishop Tuff and Glass Mountain), and also for voluminous basaltic fissure eruptions between 4 and $2 \mathrm{Ma}$ (Gilbert et al. 1968). These numerous, faulted potassic basalt flows now comprise the Adobe Hills between Mono Basin and Long Valley (Fig. 2). However, despite the perception of a silica gap between $\sim 54$ and 64 wt\% (Kelleher and Cameron 1990), volcanic activity in this region has not been bimodal, and andesite lavas also occur. There is a structural control on their eruption, and intermediate magmas predominate along the northern margin of the Mono Basin in the Aurora volcanic field, which has been active over the last 3.6 Ma, with the last eruption (hornblende andesite) occurring $110 \mathrm{ka}$ (Lange et al. 1993).

The Aurora volcanic field records a continuum of lava types from basalt through dacite. The lavas are all highly potassic and follow a classic calc-alkaline differentiation trend. A high-silica rhyolite complex also occurs and is distinguished from the Quaternary rhyolites of the Mono Craters ( $\sim 40 \mathrm{~km}$ south of Aurora) by its 3.6 Ma age. The potassic character of all Aurora lavas suggests a close genetic relationship among them, and the goal of this study was to determine the mechanism by which differentiation occurred. Specifically, we address whether the andesite magmas evolved predominantly by (1) partial melting of granitoid basement, followed by basaltic recharge and mixing, (2) fractional crystallization in largely molten magma chambers, or (3) repeated partial melting of previous batches of mantle-derived potassic magma (i.e., partial melting of young mafic, lower crust).

\section{Tectonic setting}

The Aurora volcanic field resides within a topographically and tectonically distinct part of the Basin and Range (Fig. 2), which is referred to in the literature both as the Sierran province (Leeman
Fig. 2 Map showing the location of the Aurora volcanic field (outlined in dashed box) in relation to the Mono Basin, Adobe Hills and Long Valley. Inset map locates the Mono Basin in relation to the Sierra Nevada and the Basin and Range province. $W G B$ refers to the Western Great Basin, delineated by the Sierra Nevada batholith and the dashed line

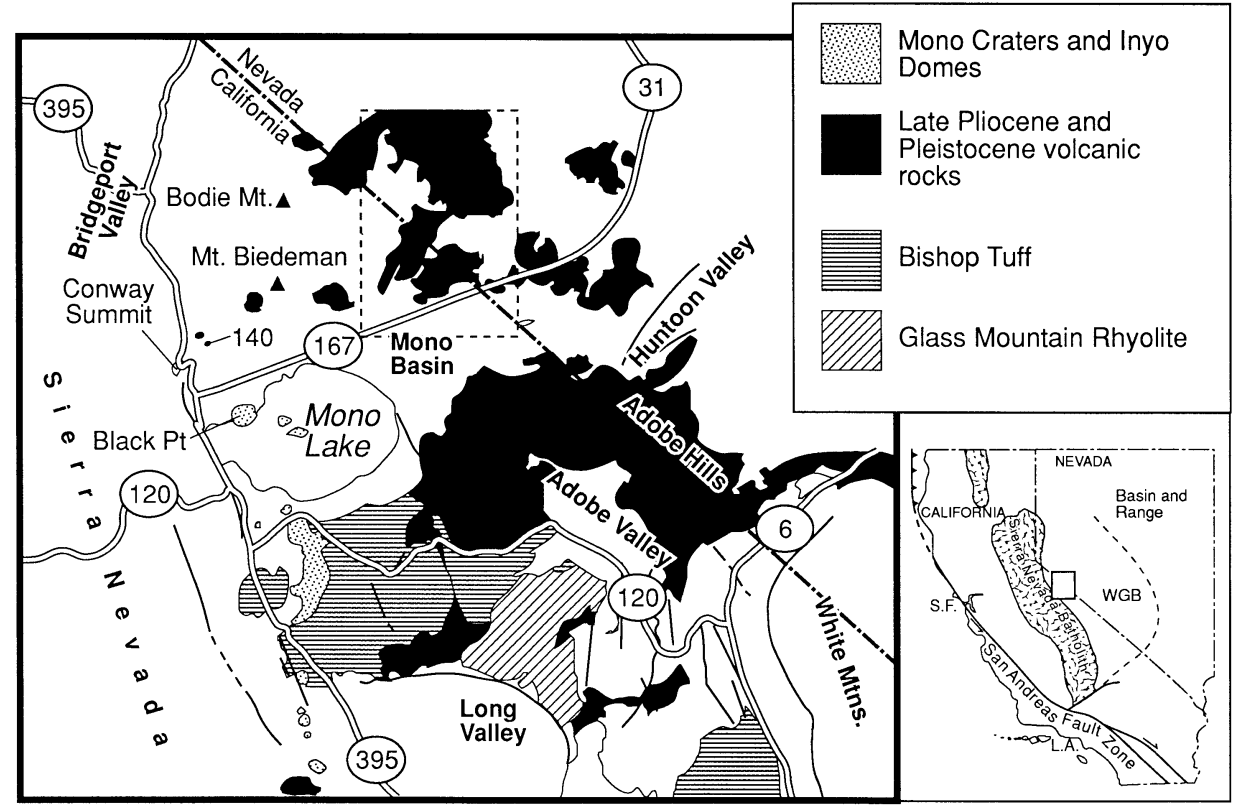


1970) and the Western Great Basin (Ormerod et al. 1988; Fitton et al. 1991). This region is defined, in part, by the anomalously high Sr isotopic values $\left({ }^{87} \mathrm{Sr} /{ }^{86} \mathrm{Sr}>0.7045\right)$ and by the potassic character of lavas within this sub-province relative to lavas from other parts of the Basin and Range (Leeman 1970; Moore and Dodge 1980; Van Kooten 1980; Ormerod et al. 1988; Feldstein and Lange 1996). It has been suggested that the source region for these potassic magmas is subduction-modified lithosphere (Kempton et al. 1991; Omerod et al. 1988; Fitton et al. 1991), which is consistent with the long history of Mesozoic and Cenozoic subduction off the west coast of North America, prior to intersection of the East Pacific Rise $\sim 30 \mathrm{Ma}$ (Atwater 1970).

\section{Eruptive history}

The oldest nonmetamorphosed rocks in the Aurora volcanic field are andesites and rhyolites that erupted between 8 and $28 \mathrm{Ma}$ (Silberman and Chesterman 1972; Silberman and McKee 1972). They overlie basement rocks of Cretaceous granitoids and metasedimentary Paleozoic units, similar to those in the Sierra Nevada (Chesterman 1968). Mineralized volcanic rocks underlie unaltered Miocene andesites near the historic mining town of Aurora; gold continues to be mined from the Aurora district.

After a hiatus between 7.5 and $4 \mathrm{Ma}$, several volcanic centers were formed and include Mt. Hicks, Beauty Peak and Aurora Peak as well as the Cedar Hill-Trench Canyon composite domes (Fig. 2). They are composed of sparse basalt and evolved $(<4 \mathrm{wt} \% \mathrm{MgO})$ basaltic andesites and andesites. Volcanic activity in the Pleistocene included the Fletcher basaltic andesite $(0.47 \mathrm{Ma})$, the Aurora Crater andesite $(0.25 \mathrm{Ma})$, and a hornblende andesite $(0.11 \mathrm{Ma})$. A summary of available K-Ar dates on Aurora lavas is presented in Table 1.

Between 3.6 and $0.1 \mathrm{Ma}, \sim 25 \mathrm{~km}^{3}$ of magma were erupted in the Aurora volcanic field, indicating an average eruption rate throughout this interval of $\sim 2 \times 10^{2} \mathrm{~m}^{3} / \mathrm{km}^{2} /$ year. This is approximately equivalent to the average eruption rate at the $1250 \mathrm{~km}^{2} \mathrm{Mt}$ Adams volcanic field in Washington over the last $900 \mathrm{ky}$, between the main cone-building pulses (i.e., "the background trickle"; Hildreth and Lanphere 1994), and is similar to the average eruption rate of the $40,000 \mathrm{~km}^{2}$ Michoacan-Guanajuato volcanic field in central Mexico over the last million years (Hasenaka 1994). The eruption rate in the Aurora volcanic field is also an order of magnitude lower than that calculated for the slow-spreading Mid-Atlantic ridge $\left(\sim 10^{3} \mathrm{~m}^{3} / \mathrm{km}^{2} /\right.$ year; BVTP 1981$)$. The relative volumes of the different Aurora lava types are: $\sim 2 \%$ basalt, $\sim 36 \%$ basaltic andesite, $\sim 16 \%$ two-pyroxene andesite, $\sim 23 \%$ hornblende andesite, $\sim 7 \%$ dacite and $\sim 16 \%$ high-silica rhyolite. These estimates of volume are based on the aerial extent of lava flows and their thickness; uncertainties are $\leq 20 \%$ relative. Thus three-quarters of the erupted magma was either basaltic andesite or andesite. There is no correlation between the age of the eruption and the composition of the flows (Table 1).

\section{Whole-rock compositions of lavas}

Sixty-two samples were collected from the various lava flows mapped in Fig. 2, and 22 were chosen for analysis on the basis of freshness and variability in modes (for modal analyses, see Table 2). Wet chemical analyses of the major elements are presented in Table 3. Trace element analyses were obtained on pressed powders (with cellulose as a binding agent) by X-ray fluorescence spectrometry (XRF) at the University of California, Berkeley; the results are shown in Table 4.

The potassic character of this suite is demonstrated in a plot of $\mathrm{K}_{2} \mathrm{O}$ vs $\mathrm{SiO}_{2}$ (Fig. 3) in which most of the samples fall either within or above the high-potassium field defined by Gill (1981); several thus fall within the shoshonitic series. Also shown in Fig. 3 are the lavas from the Adobe Hills (Lange et al. 1993; Fig. 1) that erupted along the southeastern margin of Mono Basin over the interval 4-2 Ma; they also fall within or above the high-K field. In contrast, the basaltic andesite of Black Point (Kelleher and Cameron 1990) falls within the medium-K field, as do many of the Long Valley postcaldera lavas studied by Vogel et al. (1994).

The basalt through dacite suite from the Aurora field follows a calc-alkaline trend in a plot of $\mathrm{FeO}^{\mathrm{T}} / \mathrm{MgO}$ vs $\mathrm{SiO}_{2}$ (Fig. 4). Overall, concentrations of $\mathrm{MgO}, \mathrm{CaO}$, $\mathrm{TiO}_{2}$ and $\mathrm{FeO}^{\mathrm{T}}$ follow linear decreasing trends with increasing $\mathrm{SiO}_{2}$ content (Fig. 5), indicating a prominent role for augite and titanomagnetite during differentiation. The coherency of these trends is notable given the fact that the Aurora lavas erupted over a $>$ three million year interval from separate vents and fissures (i.e., they did not erupt from a single stratovolcano). Trace element ratios such as $\mathrm{Ba} / \mathrm{Nb}(50-150)$ are typical of subductionrelated lavas; some incompatible elements (e.g., K and
Table 1 Summary of available $\mathrm{K}$-Ar ages from the Aurora volcanic field

\begin{tabular}{lllll}
\hline $\begin{array}{l}\text { Sample \# } \\
\text { (this study) }\end{array}$ & $\begin{array}{l}\text { Sample \# } \\
\text { (in reference) }\end{array}$ & Material & $\begin{array}{l}\text { Age } \pm 1 \sigma \\
(\mathrm{Ma})\end{array}$ & Reference \\
\hline 23 & 23 & Groundmass & $2.60 \pm 0.06$ & Lange et al. (1993) \\
158 & 158 & Groundmass & $0.11 \pm 0.08$ & Lange et al. (1993) \\
160 & 160 & Groundmass & $0.47 \pm 0.19$ & Lange et al. (1993) \\
162 & $672-2$ & Groundmass & $0.25 \pm 0.05$ & Silbermann and McKee (1972) \\
364 & $611-5$ & Whole-rock & $2.2 \pm 0.1$ & Silbermann and McKee (1972) \\
161 & $610-1 B$ & Biotite & $2.5 \pm 0.2$ & Silbermann and McKee (1972) \\
370 & $7346-1$ & Whole-rock & $2.7 \pm 0.1$ & Silbermann and Chesterman (1972) \\
344 & KA-2004 & Biotite & $2.5 \pm 0.1$ & Gilbert et al. (1968) \\
326 & KA-1999 & Plagioclase & $1.0 \pm 0.1$ & Gilbert et al. (1968) \\
31 & KA-1984 & Biotite & $3.4 \pm 0.3$ & Gilbert et al. (1968) \\
324 & KA-2003 & Plagioclase & $2.7 \pm 0.8$ & Gilbert et al. (1968) \\
63 & KA-1859 & Hornblende & $2.8 \pm 0.4$ & Gilbert et al. (1968) \\
334 & KA-2066 & Sanidine & $3.6 \pm 0.1$ & Gilbert et al. (1968) \\
347 & KA-2086 & Whole-rock & $2.8 \pm 0.1$ & Gilbert et al. (1968) \\
303 & KA-2089 & Whole-rock & $1.6 \pm 0.1$ & Gilbert et al. (1968) \\
203 & KA-2076 & Plagioclase & $2.6 \pm 0.4$ & Gilbert et al. (1968) \\
\hline
\end{tabular}


Table 2 Modal analyses $(>1500$ points counted) $(p h$ phenocrysts $(>0.5 \mathrm{~mm}), \mathrm{mp}$ microphenocrysts $(>0.2 \mathrm{~mm})$ ol olivine, plag plagioclase, cpx clinopyroxene, opx orthopyroxene, $h b$ hornblende, biot biotite, $q z$ quartz, $o x$ Fe-Ti oxides, gms groundmass)

\begin{tabular}{|c|c|c|c|c|c|c|c|c|c|c|c|c|}
\hline & & $\mathrm{mp}$ & - & 2.5 & - & - & 0.8 & - & - & - & - & - \\
\hline & 368 & $\mathrm{ph}$ & - & 2.4 & - & - & 0.5 & - & - & - & 5.8 & 94.2 \\
\hline & & $\mathrm{mp}$ & - & 2.0 & 0.1 & - & 0.8 & - & - & - & - & - \\
\hline & 26 & $\mathrm{ph}$ & 0.4 & 5.2 & 1.5 & - & - & - & - & - & 17.2 & 82.8 \\
\hline & & $\mathrm{mp}$ & 1.8 & 7.2 & 1.1 & - & - & - & - & - & - & - \\
\hline & 311 & $\mathrm{ph}$ & 0.4 & - & - & - & - & - & - & - & 0.8 & 99.2 \\
\hline & & $\mathrm{mp}$ & 0.4 & - & - & - & - & - & - & - & - & - \\
\hline & 160 & $\mathrm{ph}$ & 1.8 & - & - & - & - & - & - & - & 2.0 & 98.0 \\
\hline & & $\mathrm{mp}$ & 0.2 & - & - & - & - & - & - & - & - & - \\
\hline & 158 & $\mathrm{ph}$ & - & 6.3 & 0.1 & - & 1.1 & - & - & - & 11.3 & 88.7 \\
\hline & & $\mathrm{mp}$ & - & 3.2 & 0.1 & - & 0.5 & - & - & - & - & - \\
\hline & 156 & $\mathrm{ph}$ & - & 0.9 & 1.3 & Trace & 2.6 & - & - & - & 9.7 & 90.3 \\
\hline & & $\mathrm{mp}$ & - & 0.3 & 0.7 & - & 3.9 & - & - & - & - & - \\
\hline & 313 & $\mathrm{ph}$ & - & 2.1 & 2.1 & - & 2.7 & - & - & - & 10.0 & 90.0 \\
\hline & & $\mathrm{mp}$ & - & 0.5 & 0.2 & - & 2.4 & - & - & - & - & - \\
\hline & 324 & $\mathrm{ph}$ & 1.0 & 5.6 & 2.1 & - & - & - & - & - & 17.8 & 82.2 \\
\hline & & $\mathrm{mp}$ & 0.7 & 7.1 & 1.3 & - & - & - & - & - & - & - \\
\hline & 326 & $\mathrm{ph}$ & 0.4 & 15.6 & 1.2 & 0.8 & - & - & - & - & 23.5 & 76.5 \\
\hline & & $\mathrm{mp}$ & - & 1.5 & 1.0 & 2.0 & - & - & 1.0 & - & - & - \\
\hline & 162 & $\mathrm{ph}$ & Trace & - & 1.4 & 0.2 & - & - & - & - & 2.8 & 97.2 \\
\hline & & $\mathrm{mp}$ & - & - & - & 1.2 & - & - & - & - & - & - \\
\hline & 140 & $\mathrm{ph}$ & - & - & 0.2 & - & 6.9 & - & - & - & 14.3 & 85.7 \\
\hline & & $\mathrm{mp}$ & - & - & 0.1 & - & 7.1 & - & - & - & - & - \\
\hline & 328 & $\mathrm{ph}$ & - & 2.4 & 1.3 & 0.8 & - & - & - & - & 6.2 & 93.8 \\
\hline & & $\mathrm{mp}$ & - & 0.2 & 1.3 & 0.2 & - & - & - & - & - & - \\
\hline & 329 & $\mathrm{ph}$ & - & 0.2 & 1.0 & 1.2 & - & - & - & - & 4.6 & 95.4 \\
\hline & & $\mathrm{mp}$ & - & - & 2.0 & 0.2 & - & - & - & - & - & - \\
\hline & 31 & $\mathrm{ph}$ & - & 5.5 & - & - & 1.8 & 1.2 & - & - & 15.3 & 84.7 \\
\hline & & $\mathrm{mp}$ & - & 3.2 & - & - & 2.6 & 1.0 & - & - & - & - \\
\hline & 319 & $\mathrm{ph}$ & - & 2.4 & - & - & 0.8 & - & - & - & 5.7 & 94.3 \\
\hline & & $\mathrm{mp}$ & - & 1.2 & - & - & 1.3 & - & - & - & - & - \\
\hline & 25 & $\mathrm{ph}$ & - & 5.2 & - & - & 0.9 & - & - & - & 15.3 & 84.7 \\
\hline & & $\mathrm{mp}$ & - & 2.0 & - & - & 7.0 & 0.2 & - & - & - & - \\
\hline & 161 & $\mathrm{ph}$ & - & 4.7 & - & - & 0.6 & 0.8 & - & - & 15.4 & 84.6 \\
\hline ocrvets & & $\mathrm{mp}$ & - & 2.5 & - & - & 4.7 & 1.7 & 0.4 & - & - & - \\
\hline
\end{tabular}

$\mathrm{Rb}$ ) are broadly correlated with $\mathrm{SiO}_{2}$ contents, whereas others (e.g., Ba and Zr) display no such regular pattern (Fig. 5).

\section{Mineralogy}

Mineral analyses were obtained on a Cameca Camebax electron microprobe at the University of Michigan. A rastered beam $\left(9 \mu \mathrm{m}^{2}\right)$ was used for plagioclase, biotite, and hornblende to prevent alkali loss.

Olivine occurs as phenocrysts and microphenocrysts in the basalt, many of the basaltic andesites, and two of the andesites. It is the sole phenocryst in four of the basaltic andesite samples (Table 2). Phenocrysts range up to $2.0 \mathrm{~mm}$ in the basalt, but are less than $1 \mathrm{~mm}$ in the basaltic andesites $(<0.5 \mathrm{~mm}$ in sample 311$)$. Representative microprobe analyses of phenocrysts are given in Table 5; only normal zoning is observed. Differences between individual crystal cores (in a single thin section) are small, typically $2-4 \%$ Fo, but cores and rims can vary by as much as $12 \%$ Fo (Table 5 ). The observed range for cores is $\mathrm{Fo}_{87-69}$; that for rims is $\mathrm{Fo}_{82-61}$.

The modal abundance of plagioclase is highly variable, and phenocrysts are notably absent from the single absarokite (23), four of the basaltic andesites (347, 303, $160,311)$, two samples from the Aurora Crater andesite complex $(162,329)$, and the hornblende lamprophyre (140) from Conway Summit. It is a common groundmass phase in all samples. Phenocrysts are normally zoned in the basaltic andesites (sample 26, 324), and oscillatory zoned in the andesites. Only two andesites (sample 368 and 313) contain reversely zoned plagioclase grains; these have sodic cores $\left(\mathrm{An}_{28}\right.$ and $\mathrm{An}_{40}$ respectively) that appear dusty and sieve-like and are rimmed by thin, clear rims of $\mathrm{An}_{60}$ and $\mathrm{An}_{54}$ composition respectively. Typically, core and rim compositions of the oscillatory zoned plagioclase grains range from $\mathrm{An}_{65}$ to $\mathrm{An}_{45}$ (Table 6).

Augite occurs as phenocrysts and microphenocrysts in many of the samples; exceptions include four basaltic 
Table 3 Wet chemical whole-rock analyses ${ }^{\mathrm{a}}$

\begin{tabular}{|c|c|c|c|c|c|c|c|c|c|c|c|}
\hline & SN-23 & SN-347 & SN-303 & SN-306 & SN-368 & SN-26 & SN-311 & SN-160 & SN-158 & SN-156 & SN-313 \\
\hline $\mathrm{SiO}_{2}$ & 47.96 & 52.75 & 54.37 & 55.89 & 55.91 & 56.52 & 56.63 & 56.78 & 57.01 & 57.55 & 57.64 \\
\hline $\mathrm{TiO}_{2}$ & 1.49 & 1.45 & 1.34 & 1.26 & 1.30 & 0.98 & 1.17 & 1.05 & 0.97 & 0.97 & 0.96 \\
\hline $\mathrm{Al}_{2} \mathrm{O}_{3}$ & 16.31 & 17.12 & 17.43 & 17.50 & 17.50 & 17.00 & 17.63 & 17.09 & 16.91 & 16.89 & 16.90 \\
\hline $\mathrm{Fe}_{2} \mathrm{O}_{3}$ & 2.90 & 2.63 & 2.38 & 1.99 & 2.54 & 1.64 & 2.19 & 2.27 & 1.91 & 2.19 & 2.21 \\
\hline $\mathrm{FeO}$ & 5.00 & 5.33 & 4.44 & 4.70 & 4.03 & 4.53 & 4.20 & 4.48 & 4.10 & 3.96 & 3.84 \\
\hline $\mathrm{MnO}$ & 0.15 & 0.13 & 0.13 & 0.13 & 0.13 & 0.10 & 0.12 & 0.11 & 0.12 & 0.11 & 0.12 \\
\hline $\mathrm{MgO}$ & 7.68 & 5.37 & 3.64 & 3.33 & 3.34 & 3.93 & 3.20 & 3.87 & 3.79 & 3.93 & 3.74 \\
\hline $\mathrm{CaO}$ & 10.06 & 7.27 & 5.94 & 5.57 & 5.70 & 6.47 & 5.78 & 6.29 & 6.10 & 5.85 & 5.94 \\
\hline $\mathrm{Na}_{2} \mathrm{O}$ & 3.06 & 4.10 & 4.26 & 4.32 & 4.44 & 3.82 & 4.24 & 4.17 & 4.19 & 4.01 & 4.19 \\
\hline $\mathrm{K}_{2} \mathrm{O}$ & 2.57 & 2.19 & 3.46 & 3.48 & 3.46 & 3.18 & 3.19 & 2.82 & 2.74 & 2.88 & 2.88 \\
\hline $\mathrm{P}_{2} \mathrm{O}_{5}$ & 0.99 & 0.48 & 0.80 & 0.72 & 0.70 & 0.41 & 0.57 & 0.47 & 0.44 & 0.45 & 0.45 \\
\hline $\mathrm{H}_{2} \mathrm{O}^{+}$ & 1.05 & 0.78 & 1.22 & 0.83 & 0.46 & 0.80 & 0.80 & 0.78 & 1.17 & 0.69 & 0.75 \\
\hline $\mathrm{H}_{2} \mathrm{O}^{-}$ & 0.22 & 0.17 & 0.23 & 0.15 & 0.09 & 0.19 & 0.06 & 0.16 & 0.21 & 0.12 & 0.15 \\
\hline \multirow[t]{2}{*}{ Total } & 99.44 & 99.77 & 99.64 & 99.87 & 99.60 & 99.57 & 99.78 & 100.34 & 99.66 & 99.60 & 99.77 \\
\hline & $\mathrm{SN}-324$ & SN-326 & SN-162 & SN-140 & SN-328 & SN-329 & SN-31 & $\mathrm{SN}-319$ & $\mathrm{SN}-25$ & SN-161 & SN-334 \\
\hline $\mathrm{SiO}_{2}$ & 57.71 & 58.54 & 59.04 & 59.31 & 59.54 & 59.82 & 60.25 & 61.94 & 62.81 & 64.31 & 76.85 \\
\hline $\mathrm{TiO}_{2}$ & 1.03 & 1.00 & 0.93 & 0.82 & 0.92 & 0.90 & 0.80 & 0.79 & 0.65 & 0.69 & 0.09 \\
\hline $\mathrm{Al}_{2} \mathrm{O}_{3}$ & 16.73 & 17.08 & 16.69 & 16.79 & 16.68 & 16.68 & 16.06 & 17.14 & 15.92 & 16.52 & 12.44 \\
\hline $\mathrm{Fe}_{2}^{2} \mathrm{O}_{3}$ & 1.41 & 1.80 & 1.25 & 1.99 & 1.42 & 1.38 & 2.33 & 1.99 & 1.51 & 1.64 & 0.24 \\
\hline $\mathrm{FeO}$ & 4.31 & 3.68 & 4.13 & 3.23 & 4.14 & 4.05 & 1.96 & 2.61 & 2.27 & 1.81 & 0.34 \\
\hline $\mathrm{MnO}$ & 0.10 & 0.07 & 0.10 & 0.10 & 0.11 & 0.11 & 0.07 & 0.09 & 0.07 & 0.07 & 0.04 \\
\hline $\mathrm{MgO}$ & 3.34 & 2.88 & 2.86 & 3.88 & 2.87 & 2.80 & 2.22 & 1.54 & 2.23 & 1.78 & 0.08 \\
\hline $\mathrm{CaO}$ & 5.44 & 5.41 & 5.10 & 5.98 & 5.14 & 5.08 & 4.74 & 4.32 & 4.15 & 3.42 & 0.60 \\
\hline $\mathrm{Na}_{2} \mathrm{O}$ & 3.73 & 4.13 & 4.04 & 4.35 & 4.28 & 4.22 & 3.72 & 4.26 & 4.03 & 3.90 & 4.04 \\
\hline $\mathrm{K}_{2} \mathrm{O}$ & 4.05 & 3.52 & 3.38 & 2.02 & 3.36 & 3.42 & 4.24 & 3.62 & 3.96 & 3.85 & 4.82 \\
\hline $\mathrm{P}_{2} \mathrm{O}_{5}$ & 0.55 & 0.42 & 0.41 & 0.24 & 0.43 & 0.46 & 0.38 & 0.27 & 0.34 & 0.29 & 0.01 \\
\hline $\mathrm{H}_{2} \mathrm{O}^{+}$ & 0.82 & 1.05 & 0.80 & 0.80 & 0.74 & 0.41 & 2.34 & 0.91 & 1.43 & 1.01 & 0.40 \\
\hline $\mathrm{H}_{2} \mathrm{O}^{-}$ & 0.18 & 0.09 & 0.19 & 0.05 & 0.11 & 0.14 & 0.38 & 0.19 & 0.27 & 0.32 & 0.02 \\
\hline Total & 99.42 & 99.67 & 99.57 & 99.56 & 99.74 & 99.47 & 99.49 & 99.67 & 99.64 & 99.61 & 99.95 \\
\hline
\end{tabular}

${ }^{a}$ Wet chemical analyst: ISE Carmichael

andesites and four hornblende andesites (Table 1). Representative microprobe analyses are presented in Table 7. Typically, rims are rich in Fe relative to $\mathrm{Mg}$. Reverse zoning of augite was found in sample 156, suggesting a xenocrystic origin.

Or thopyroxene (Table 8) occurs as sparse phenocrysts and microphenocrysts in all samples from the Aurora Crater center $(162,328,329)$ and in the two-pyroxene andesite flow from the Cedar Hill complex (326). A single grain of orthopyroxene was found in sample 156; although it is normally zoned, it may be xenocrystic. Pigeonite was not found in any sample.

Phenocrysts and microphenocrysts of hornblende occur in both flows from a basaltic andesite center (306, 368 ), the hornblende lamprophyre (140), the youngest hornblende andesite flow $(156,158,313)$, and four additional hornblende andesite lava domes and flows (31, $319,25,161)$. Hornblende never occurs with or thopyroxene phenocrysts, but occasionally with augite phenocrysts. In many samples, the hornblende crystals are surrounded by a fine-grained reaction rim of opacite. In other samples $(31,319$ and 161), the hornblende phenocryst grains are remarkably free of reaction rims. Representative microprobe analyses are presented in Table 9; mineral formulae were normalized about 13 small cations. For these calculations, water contents were cal- culated assuming that $\mathrm{OH}=2-(\mathrm{Cl}+\mathrm{F})$, and the $\mathrm{Fe}^{2+} /$ $\mathrm{Fe}^{3+}$ ratios were estimated from charge balance. These estimates of $\mathrm{Fe}_{2} \mathrm{O}_{3}$ and $\mathrm{H}_{2} \mathrm{O}$ presented in Table 9 should be viewed as minimum and maximum values respectively (Cosca et al. 1991). Hornblende separates (free of any alteration and reaction rims) from samples $\mathrm{SN}-31$ and SN-161 were analyzed for $\mathrm{FeO}$ by wet chemical techniques and for $\mathrm{H}_{2} \mathrm{O}$ by manometry (Feldstein et al. 1996). Core $\mathrm{Fe}^{3+} / \Sigma \mathrm{Fe}$ values in samples 31 and 161 are 0.56 and 0.61 respectively, similar to values obtained on hornblende from calc-alkaline granodiorites (Speer 1984). F contents are low $(\leq 0.3 \mathrm{wt} \%)$ in all samples.

Sparse biotite phenocrysts and microphenocrysts occur in three hornblende andesite flows and domes $(31,25$ and 161); analyses are given in Table 10. Pure biotite separates (free of reaction rims) were analyzed for $\mathrm{FeO}$ by wet chemistry and $\mathrm{H}_{2} \mathrm{O}$ by manometry (Feldstein et al. 1996). Core $\mathrm{Fe}^{3+} / \Sigma \mathrm{Fe}$ ratios in samples 31 and 161 are 0.56 and 0.66 , similar to those observed in the hornblende separates from the same samples and values found in biotites from calc-alkaline granodiorites (Speer 1984). As seen for the hornblende phenocrysts, fluorine contents in the biotites are low $(\leq 0.15 \mathrm{wt} \%)$.

$\mathrm{Cr}$-spinel inclusions in olivine (Table 11) occur in all samples that contain olivine phenocrysts; $\mathrm{Fe}^{3+} / \Sigma \mathrm{Fe}$ ra- 
Table 4 XRF trace element whole-rock analyses ${ }^{\mathrm{a}}(b . d$. below detection limit)

\begin{tabular}{|c|c|c|c|c|c|c|c|c|c|c|c|}
\hline & $\mathrm{SN}-23$ & SN-347 & SN-303 & SN-306 & SN-368 & SN-26 & SN-311 & SN-160 & SN-158 & SN-156 & SN-313 \\
\hline $\mathrm{V}$ & 127 & 160 & 142 & 130 & 135 & 124 & 133 & 81 & 144 & 126 & 110 \\
\hline $\mathrm{Cr}$ & 174 & 80 & 57 & 51 & 61 & 58 & 51 & 10 & 83 & 83 & 53 \\
\hline $\mathrm{Ni}$ & 115 & 65 & 27 & 16 & 30 & 33 & 27 & 32 & 37 & 33 & 43 \\
\hline $\mathrm{Cu}$ & 43 & 28 & 19 & 61 & 49 & 33 & 35 & 31 & 35 & 29 & 39 \\
\hline $\mathrm{Zn}$ & 72 & 87 & 91 & 84 & 84 & 77 & 90 & 83 & 89 & 84 & 89 \\
\hline $\mathrm{Rb}$ & 38 & 33 & 71 & 70 & 71 & 56 & 63 & 44 & 42 & 44 & 46 \\
\hline $\mathrm{Sr}$ & 1780 & 1110 & 1100 & 1025 & 1072 & 1035 & 980 & 1010 & 1085 & 1060 & 1075 \\
\hline $\mathrm{Y}$ & 26 & 25 & 27 & 27 & 27 & 22 & 25 & 25 & 24 & 23 & 22 \\
\hline $\mathrm{Zr}$ & 243 & 210 & 345 & 320 & 330 & 239 & 325 & 289 & 271 & 262 & 275 \\
\hline $\mathrm{Nb}$ & 19 & 17 & 31 & 29 & 31 & 17 & 27 & 20 & 22 & 18 & 17 \\
\hline $\mathrm{Ba}$ & 1830 & 1155 & 1815 & 1565 & 1620 & 1550 & 1470 & 1590 & 1550 & $1550 ?$ & 1315 \\
\hline $\mathrm{La}$ & 81 & 41 & 61 & 58 & 62 & 45 & 53 & 55 & 51 & $51 ?$ & 49 \\
\hline \multirow[t]{2}{*}{$\mathrm{Ce}$} & 161 & 76 & 130 & 120 & 130 & 97 & 113 & 100 & 101 & $101 ?$ & 92 \\
\hline & SN-324 & SN-326 & SN-162 & SN-140 & SN-328 & SN-329 & $\mathrm{SN}-31$ & SN-319 & SN-25 & SN-161 & SN-334 \\
\hline V & 120 & 110 & 119 & 108 & 107 & 104 & 52 & 85 & 65 & 81 & 0.3 \\
\hline $\mathrm{Cr}$ & 38 & 33 & 34 & 102 & 31 & 39 & 9 & 56 & 10 & b.d. & b.d. \\
\hline $\mathrm{Ni}$ & 45 & 26 & 33 & 74 & 30 & 28 & 14 & 12 & 31 & 22 & 26 \\
\hline $\mathrm{Cu}$ & 29 & 31 & 26 & 27 & 23 & 34 & 14 & 20 & 20 & 16 & 9 \\
\hline $\mathrm{Zn}$ & 79 & 65 & 86 & 79 & 87 & 98 & 55 & 69 & 62 & 52 & 22 \\
\hline $\mathrm{Rb}$ & 90 & 72 & 45 & 45 & 55 & 58 & 89 & 71 & 76 & 83 & 152 \\
\hline $\mathrm{Sr}$ & 855 & 1145 & 765 & 1025 & 780 & 788 & 940 & 790 & 930 & 965 & 22 \\
\hline $\mathrm{Y}$ & 23 & 19 & 27 & 17 & 25 & 24 & 21 & 19 & 19 & 18 & 13 \\
\hline $\mathrm{Zr}$ & 365 & 255 & 342 & 133 & 365 & 365 & 266 & 210 & 254 & 243 & 87 \\
\hline $\mathrm{Nb}$ & 26 & 14 & 23 & 9 & 19 & 19 & 14 & 12 & 13 & 15 & 20 \\
\hline $\mathrm{Ba}$ & 1645 & 1555 & 1725 & 1145 & 1495 & 1490 & 2085 & 1520 & 1965 & 2040 & 55 \\
\hline $\mathrm{La}$ & 58 & 46 & 63 & 26 & 52 & 55 & 54 & 35 & 40 & 51 & 29 \\
\hline $\mathrm{Ce}$ & 114 & 100 & 112 & 66 & 108 & 110 & - & 62 & - & 95 & 51 \\
\hline
\end{tabular}

${ }^{\text {a }}$ One standard deviation errors are equal to the following percentages of the amount present: $\mathrm{V}(8 \%), \mathrm{Cr}(10 \%), \mathrm{Ni}(15 \%), \mathrm{Cu}(12 \%)$ $\mathrm{Zn}(10 \%), \mathrm{Rb}(10 \%), \mathrm{Sr}(5 \%), \mathrm{Y}(15 \%), \mathrm{Zr}(5 \%), \mathrm{Nb}(10 \%), \mathrm{Ba}(3 \%), \mathrm{La}(15 \%)$, and $\mathrm{Ce}(10 \%)$

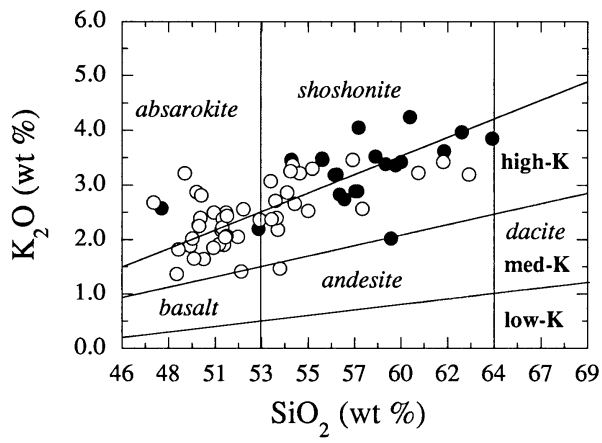

Fig. 3 A plot of $\mathrm{K}_{2} \mathrm{O}$ vs $\mathrm{SiO}_{2}$ for lavas from the Aurora volcanic field (solid circles) and the Adobe Hills (open circles). Most lavas fall within the high-K or shoshonitic field. Boundaries defining the low-K, medium-K and high-K fields are from Gill (1981)

tios range from 0.41 to 0.56 . The chromites display significant solid solution with $\mathrm{Fe}_{3} \mathrm{O}_{4}$ and $\mathrm{Fe}_{2} \mathrm{TiO}_{4}$ in samples 303, 311 and 160. Titanomagnetite does not occur as a microphenocryst or phenocryst in any lava, but is a ubiquitous groundmass phase (Table 12), and is often quite coarse in the andesites. The $\mathrm{Fe}_{3} \mathrm{O}_{4}$ component $\left(X_{\text {mte }}\right)$ varies between 0.40 to 0.78 , whereas the $\mathrm{Fe}^{3+} /$ $\Sigma$ Fe ratio ranges from 0.38 to 0.59 . Ilmenite does not occur in any sample.

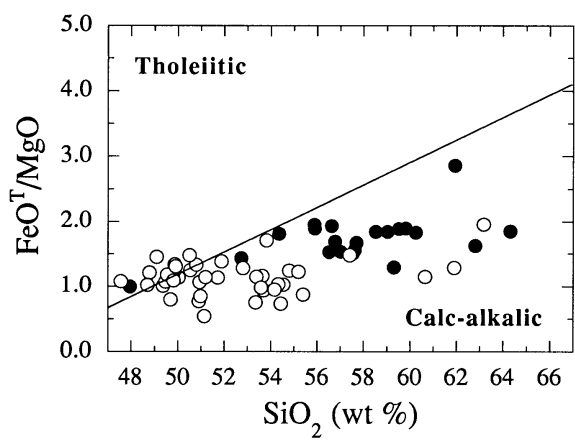

Fig. 4 A plot of $\mathrm{FeO}^{\mathrm{T}} / \mathrm{MgO}$ vs $\mathrm{SiO}_{2}$ for lavas from the Aurora volcanic field (solid circles) and the Adobe Hills (open circles). The differentiation trend defined by all lavas is calc-alkaline. The boundary between the tholeiitic and calc-alkalic fields is from Miyashiro (1974)

\section{Intensive variables}

\section{Temperature}

Fe-Ti oxide thermometry could not be applied because of the absence of ilmenite in all samples. Temperatures were calculated, however, for those andesites that contain both orthopyroxene and augite phenocrysts (sam- 
Table 5 Olivine phenocryst analyses (b.d. below detection limit)

\begin{tabular}{|c|c|c|c|c|c|c|c|c|c|c|c|c|c|c|}
\hline & $\begin{array}{l}\text { SN-23 } \\
\text { Core }\end{array}$ & $\begin{array}{l}\text { SN-23 } \\
\text { Rim }\end{array}$ & $\begin{array}{l}\text { SN-347 } \\
\text { Core }\end{array}$ & $\begin{array}{l}\text { SN-347 } \\
\text { Rim }\end{array}$ & $\begin{array}{l}\text { SN-303 } \\
\text { Core }\end{array}$ & $\begin{array}{l}\text { SN-303 } \\
\text { Rim }\end{array}$ & $\begin{array}{l}\text { SN-26 } \\
\text { Core }\end{array}$ & $\begin{array}{l}\text { SN-26 } \\
\text { Rim }\end{array}$ & $\begin{array}{l}\text { SN-311 } \\
\text { Core }\end{array}$ & $\begin{array}{l}\text { SN-311 } \\
\text { Rim }\end{array}$ & $\begin{array}{l}\text { SN-160 } \\
\text { Core }\end{array}$ & $\begin{array}{l}\text { SN-160 } \\
\text { Rim }\end{array}$ & $\begin{array}{l}\mathrm{SN}-324 \\
\text { Core }\end{array}$ & $\begin{array}{l}\text { SN-324 } \\
\text { Rim }\end{array}$ \\
\hline $\mathrm{SiO}_{2}$ & 38.3 & 39.1 & 38.0 & 36.4 & 37.2 & 38.4 & 36.8 & 37.0 & 37.4 & 37.3 & 36.8 & 35.6 & 36.6 & 36.9 \\
\hline $\mathrm{Al}_{2} \mathrm{O}_{3}$ & 0.08 & 0.07 & 0.01 & 0.02 & b.d. & 0.01 & 0.01 & 0.03 & b.d. & 0.04 & 0.02 & 0.04 & b.d. & 0.02 \\
\hline $\mathrm{Cr}_{2} \mathrm{O}_{3}$ & 0.32 & 0.29 & 0.01 & b.d. & b.d. & 0.01 & b.d. & 0.01 & 0.02 & 0.03 & 0.01 & 0.04 & b.d. & b.d. \\
\hline $\mathrm{FeO}$ & 13.3 & 14.3 & 18.2 & 24.2 & 20.9 & 20.6 & 27.8 & 28.3 & 22.00 & 23.9 & 25.1 & 30.3 & 28.6 & 28.9 \\
\hline $\mathrm{MnO}$ & 0.36 & 0.39 & 0.23 & 0.53 & 0.34 & 0.34 & 0.48 & 0.60 & 0.58 & 0.62 & 0.50 & 0.66 & 0.58 & 0.50 \\
\hline $\mathrm{NiO}$ & 0.48 & 0.44 & 0.19 & 0.14 & 0.15 & 0.08 & 0.11 & 0.11 & 0.10 & 0.03 & 0.10 & 0.05 & 0.10 & 0.09 \\
\hline $\mathrm{MgO}$ & 46.1 & 44.6 & 43.6 & 37.2 & 40.7 & 39.9 & 35.4 & 33.8 & 39.9 & 37.6 & 37.0 & 32.3 & 34.6 & 34.1 \\
\hline $\mathrm{CaO}$ & 0.19 & 0.23 & 0.13 & 0.53 & 0.20 & 0.17 & 0.23 & 0.22 & 0.15 & 0.24 & 0.17 & 0.20 & 0.29 & 0.26 \\
\hline Total & 99.1 & 99.5 & 100.3 & 99.0 & 99.5 & 99.6 & 100.9 & 100.1 & 100.2 & 99.7 & 99.7 & 99.1 & 100.7 & 100.7 \\
\hline$X_{\mathrm{Mg}}$ & 0.87 & 0.84 & 0.83 & 0.74 & 0.79 & 0.77 & 0.70 & 0.68 & 0.78 & 0.74 & 0.73 & 0.66 & 0.69 & 0.68 \\
\hline$X_{\mathrm{Fe}}$ & 0.14 & 0.15 & 0.19 & 0.27 & 0.23 & 0.22 & 0.31 & 0.32 & 0.24 & 0.26 & 0.28 & 0.35 & 0.32 & 0.32 \\
\hline P.R. ${ }^{a}$ & $87-82$ & & $83-73$ & & $79-77$ & & $70-68$ & & $78-73$ & & $73-61$ & & $69-67$ & \\
\hline
\end{tabular}

${ }^{a}$ Phenocryst range (core and rim)

Table 6 Plagioclase phenocryst analyses

\begin{tabular}{|c|c|c|c|c|c|c|c|c|c|c|c|c|c|c|c|c|c|c|}
\hline & $\begin{array}{l}319 \\
\text { Core }\end{array}$ & $\begin{array}{l}319 \\
\text { Rim }\end{array}$ & $\begin{array}{l}31 \\
\text { Core }\end{array}$ & $\begin{array}{l}31 \\
\text { Rim }\end{array}$ & $\begin{array}{l}161 \\
\text { Core }\end{array}$ & $\begin{array}{l}161 \\
\text { Rim }\end{array}$ & $\begin{array}{l}25 \\
\text { Core }\end{array}$ & $\begin{array}{l}25 \\
\text { Rim }\end{array}$ & $\begin{array}{l}328 \\
\text { Core }\end{array}$ & $\begin{array}{l}328 \\
\operatorname{Rim}\end{array}$ & $\begin{array}{l}326 \\
\text { Core }\end{array}$ & $\begin{array}{l}326 \\
\text { Rim }\end{array}$ & $\begin{array}{l}26 \\
\text { Core }\end{array}$ & $\begin{array}{l}26 \\
\text { Rim }\end{array}$ & $\begin{array}{l}368 \\
\text { Core }\end{array}$ & $\begin{array}{l}368 \\
\text { Rim }\end{array}$ & $\begin{array}{l}156 \\
\text { Core }\end{array}$ & $\begin{array}{l}156 \\
\text { Rim }\end{array}$ \\
\hline $\mathrm{O}$ & 50.0 & 52.4 & 52.3 & 53.5 & 54.4 & 56.6 & 53.5 & 54.6 & 52.1 & 57.3 & 53.6 & 56.0 & 50.7 & 55.6 & 58.78 & 50.72 & 55.17 & 52.37 \\
\hline${ }_{2} \mathrm{O}_{3}$ & 30.6 & 29.4 & 29.6 & 28.9 & 27.9 & 26.8 & 29.2 & 28.4 & 29.2 & 25.8 & 28.2 & 26.7 & 30.0 & 26.3 & 24.65 & 29.79 & 27.86 & 28.53 \\
\hline $\mathrm{Fe}_{2} \mathrm{O}_{3}$ & 0.78 & 0.77 & 0.71 & 0.64 & 0.57 & 0.62 & 0.59 & 0.73 & 0.92 & 1.10 & 0.71 & 0.82 & 0.94 & 1.06 & 0.43 & 0.97 & 0.59 & 1.02 \\
\hline $\mathrm{CaO}$ & 14.0 & 12.1 & 11.9 & 10.9 & 10.2 & 8.8 & 11.6 & 10.7 & 12.7 & 8.6 & 11.2 & 9.8 & 13.2 & 8.9 & 5.80 & 12.08 & 8.93 & 11.01 \\
\hline $\mathrm{SrC}$ & 0.39 & 0.40 & 0.46 & 0.45 & 0.52 & 0.54 & 0.49 & 0.50 & 0.41 & 0.37 & 0.46 & 0.44 & 0.41 & 0.40 & 0.29 & 0.54 & 0.29 & 0.52 \\
\hline $\mathrm{BaO}$ & 0.22 & 0.22 & 0.24 & 0.23 & 0.23 & 0.27 & 0.24 & 0.27 & 0.24 & 0.28 & 0.29 & 0.28 & 0.22 & 0.26 & 0.47 & 0.45 & 0.24 & 0.26 \\
\hline $\mathrm{Na}_{2} \mathrm{O}$ & 3.28 & 4.18 & 4.17 & 4.74 & 5.19 & 5.81 & 4.27 & 4.71 & 3.66 & 5.47 & 4.34 & 4.95 & 3.65 & 5.66 & 7.09 & 4.27 & 5.83 & 4.83 \\
\hline $\mathrm{K}_{2} \mathrm{O}$ & 0.27 & 0.40 & 0.42 & 0.53 & 0.50 & 0.60 & 0.43 & 0.56 & 0.47 & 0.95 & 0.63 & 0.88 & 0.46 & 0.94 & 1.08 & 0.36 & 0.38 & 0.59 \\
\hline Total & 99.4 & 99.8 & 99.8 & 99.9 & 99.5 & 100.0 & 100.3 & 100.4 & 99.7 & 99.8 & 99.4 & 99.9 & 99.6 & 99.1 & 98.59 & 99.18 & 99.29 & 99.13 \\
\hline$X_{\mathrm{C} z}$ & 0.69 & 0.60 & 0.59 & 0.53 & 0.50 & 0.43 & 0.57 & 0.52 & 0.63 & 0.42 & 0.55 & 0.48 & 0.66 & 0.44 & 0.283 & 0.600 & 0.440 & 0.543 \\
\hline$X_{1}$ & 0 & 0.3 & 0.37 & . & & 0.47 & 0.3 & 0.42 & 0.33 & 0.48 & 0.39 & 0. & 0.33 & 0.51 & 0.6 & & & \\
\hline$X_{\mathrm{K}}$ & 0.02 & 0.02 & 0.03 & 0.03 & 0.03 & 0.04 & 0.03 & 0.03 & 0.03 & 0.06 & 0.04 & 0.05 & 0.03 & 0.06 & 0.063 & 30.021 & 10.022 & 20.035 \\
\hline P.R. ${ }^{a}$ & $69-5$ & & $61-5$ & & 5 & & 57 & & 67 & & 61 & & 66 & & 63 & & 62 & \\
\hline R.R. ${ }^{\mathrm{b}}$ & $64-54$ & & $57-50$ & & $55-43$ & & $56-49$ & & $59-41$ & & $57-33$ & & $50-44$ & & $62-58$ & & $61-54$ & \\
\hline
\end{tabular}

${ }^{a}$ Range in phenocryst composition (core and rim; mole \% anorthite)

${ }^{\mathrm{b}}$ Range in rim composition (mole \% anorthite)

Table 7 Clinopyroxene phenocryst analyses

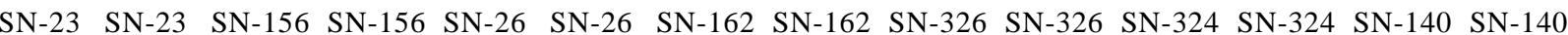
Core Rim Core Rim Core Rim Core Rim Core Rim Core Rim Core Rim

\begin{tabular}{lccccccccccccccc}
\hline $\mathrm{SiO}_{2}$ & 51.2 & 48.5 & 50.6 & 51.8 & 51.0 & 50.9 & 51.7 & 50.3 & 51.5 & 50.49 & 50.5 & 50.8 & 52.9 & 52.6 \\
$\mathrm{TiO}_{2}$ & 0.62 & 1.10 & 0.76 & 0.63 & 1.00 & 0.98 & 0.75 & 0.81 & 0.77 & 0.81 & 0.86 & 0.88 & 0.27 & 0.25 \\
$\mathrm{Al}_{2} \mathrm{O}_{3}$ & 4.27 & 6.22 & 4.67 & 3.44 & 2.26 & 2.52 & 2.26 & 3.04 & 2.49 & 4.19 & 4.01 & 2.48 & 1.73 & 2.03 \\
$\mathrm{Cr}_{2} \mathrm{O}_{3}$ & 0.64 & 0.87 & 0.80 & 1.00 & 0.40 & 0.46 & 0.42 & 0.53 & 0.05 & 0.11 & 0.16 & 0.12 & 0.01 & 0.05 \\
$\mathrm{FeO}$ & 4.78 & 5.20 & 7.03 & 6.42 & 9.62 & 10.10 & 7.68 & 9.74 & 7.98 & 7.88 & 7.45 & 9.06 & 5.94 & 6.41 \\
$\mathrm{MnO}$ & 0.15 & 0.15 & 0.31 & 0.35 & 0.34 & 0.36 & 0.36 & 0.41 & 0.17 & 0.14 & 0.17 & 0.27 & 0.19 & 0.12 \\
$\mathrm{MgO}$ & 16.1 & 14.2 & 15.1 & 16.1 & 15.8 & 15.5 & 16.0 & 14.9 & 15.5 & 15.3 & 16.2 & 15.2 & 16.4 & 16.1 \\
$\mathrm{CaO}$ & 21.5 & 22.9 & 20.1 & 20.3 & 18.8 & 18.4 & 21.2 & 20.8 & 20.8 & 20.4 & 19.2 & 19.7 & 21.9 & 21.8 \\
$\mathrm{Na} 2$ & 0.50 & 0.43 & 0.83 & 0.63 & 0.47 & 0.44 & 0.41 & 0.48 & 0.38 & 0.47 & 0.39 & 0.36 & 0.39 & 0.45 \\
$\mathrm{Total}$ & 99.7 & 99.6 & 100.3 & 100.7 & 99.7 & 99.7 & 100.8 & 100.9 & 99.6 & 99.8 & 98.9 & 98.8 & 99.8 & 99.8 \\
& & & & & & & & & & & & & & \\
$X_{\text {En }}$ & 0.44 & 0.39 & 0.42 & 0.44 & 0.44 & 0.43 & 0.44 & 0.41 & 0.43 & 0.42 & 0.45 & 0.43 & 0.45 & 0.44 \\
$X_{\mathrm{Fs}}$ & 0.07 & 0.08 & 0.11 & 0.10 & 0.15 & 0.16 & 0.12 & 0.15 & 0.12 & 0.12 & 0.12 & 0.14 & 0.09 & 0.10 \\
$X_{\text {wo }}$ & 0.42 & 0.46 & 0.40 & 0.40 & 0.38 & 0.37 & 0.42 & 0.41 & 0.41 & 0.41 & 0.38 & 0.40 & 0.43 & 0.43 \\
\hline
\end{tabular}


Fig. 5a, b $\mathrm{SiO}_{2}$ variation diagrams showing abundances of selected major and trace elements for 21 lavas from the Aurora volcanic field. a $\mathrm{CaO}$ $\mathrm{FeO}^{\mathrm{T}}, \mathrm{TiO}_{2}$ and $\mathrm{MgO}$ follow strongly linear decreasing trends with $\mathrm{SiO}_{2}$ content b The strongly incompatible elements, $\mathrm{Ba}$ and $\mathrm{Zr}$, show complete scatter with $\mathrm{SiO}_{2}$ content
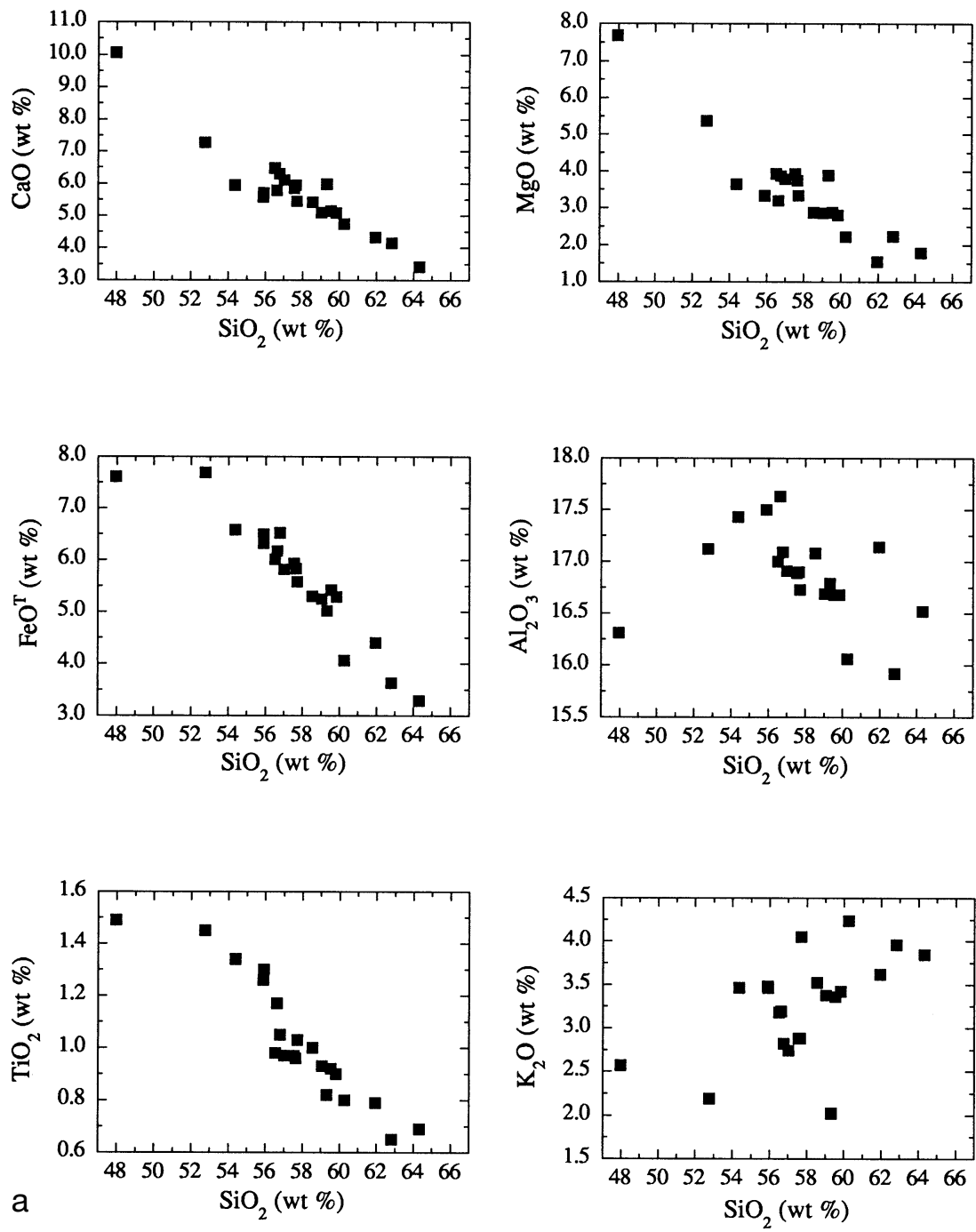

Table 8 Orthopyroxene phenocryst analyses

\begin{tabular}{|c|c|c|c|c|c|c|}
\hline & $\begin{array}{l}\text { SN-156 } \\
\text { Core }\end{array}$ & $\begin{array}{l}\text { SN-156 } \\
\text { Rim }\end{array}$ & $\begin{array}{l}\text { SN-326 } \\
\text { Core }\end{array}$ & $\begin{array}{l}\text { SN-326 } \\
\text { Rim }\end{array}$ & $\begin{array}{l}\text { SN-162 } \\
\text { Core }\end{array}$ & $\begin{array}{l}\text { SN-162 } \\
\text { Rim }\end{array}$ \\
\hline $\mathrm{SiO}_{2}$ & 52.8 & 52.6 & 53.3 & 53.6 & 53.1 & 52.6 \\
\hline $\mathrm{TiO}_{2}$ & 0.22 & 0.27 & 0.37 & 0.32 & 0.18 & 0.48 \\
\hline $\mathrm{Al}_{2} \mathrm{O}_{3}$ & 2.76 & 3.46 & 1.32 & 1.12 & 1.84 & 1.25 \\
\hline $\mathrm{Cr}_{2} \mathrm{O}_{3}$ & 0.30 & 0.17 & b.d. & b.d. & 0.03 & 0.31 \\
\hline $\mathrm{FeO}$ & 12.2 & 13.4 & 6.8 & 16.0 & 15.3 & 17.7 \\
\hline $\mathrm{MnO}$ & 0.27 & 0.28 & 0.47 & 0.49 & 0.37 & 0.68 \\
\hline $\mathrm{MgO}$ & 28.4 & 27.0 & 25.8 & 26.4 & 27.4 & 24.9 \\
\hline $\mathrm{CaO}$ & 1.40 & 1.70 & 1.38 & 1.48 & 1.49 & 1.85 \\
\hline $\mathrm{Na}_{2} \mathrm{O}$ & 0.06 & 0.06 & 0.03 & 0.02 & 0.04 & 0.10 \\
\hline Total & 98.4 & 99.0 & 99.5 & 99.5 & 99.8 & 99.9 \\
\hline$X_{\text {En }}$ & 0.77 & 0.73 & 0.71 & 0.72 & 0.74 & 0.68 \\
\hline$X_{\mathrm{Fs}}$ & 0.19 & 0.20 & 0.26 & 0.24 & 0.23 & 0.27 \\
\hline$X_{\mathrm{Wo}}$ & 0.03 & 0.03 & 0.03 & 0.03 & 0.03 & 0.04 \\
\hline
\end{tabular}

ples 162, 156 and 326), using the thermometer of Lindsley and Frost (QUILF; 1992). Temperatures of $1067 \pm 5^{\circ} \mathrm{C}$ (sample 162), $1079 \pm 5^{\circ} \mathrm{C}$ (sample 156) and $1041 \pm 31^{\circ} \mathrm{C}$ (sample 326 ), assuming equilibration at 1-3 kbar, were obtained. Because the augite is reversely zoned in sample 156 and only a single grain of orthopyroxene was observed in one of several thin sections from this flow, the temperature of $1079 \pm 5^{\circ} \mathrm{C}$ probably represents that of a disaggregated xenolith and not necessarily that of the magma itself.

Temperatures can also be estimated for those lavas containing biotite using the thermometer of Righter and Carmichael (1996) based on the partitioning of $\mathrm{TiO}_{2}$ between biotite and liquid. Application of this thermometer requires that the $\mathrm{TiO}_{2}$ content of the groundmass be known; electron microprobe analyses were performed in raster mode and by continuously moving the sample during the counting time (Table 13). From the data in Tables 10 and 13 , samples 25,31 , and 161 have calculated temperatures of 951,937 , and $938^{\circ} \mathrm{C}$ respectively. These temperatures are more than 100 degrees cooler than those inferred for the two-pyroxene andesites without any hydrous phenocrysts, suggesting that the biotitebearing andesites, in addition to being more potassic, contained significantly more water. 
Fig. 5b
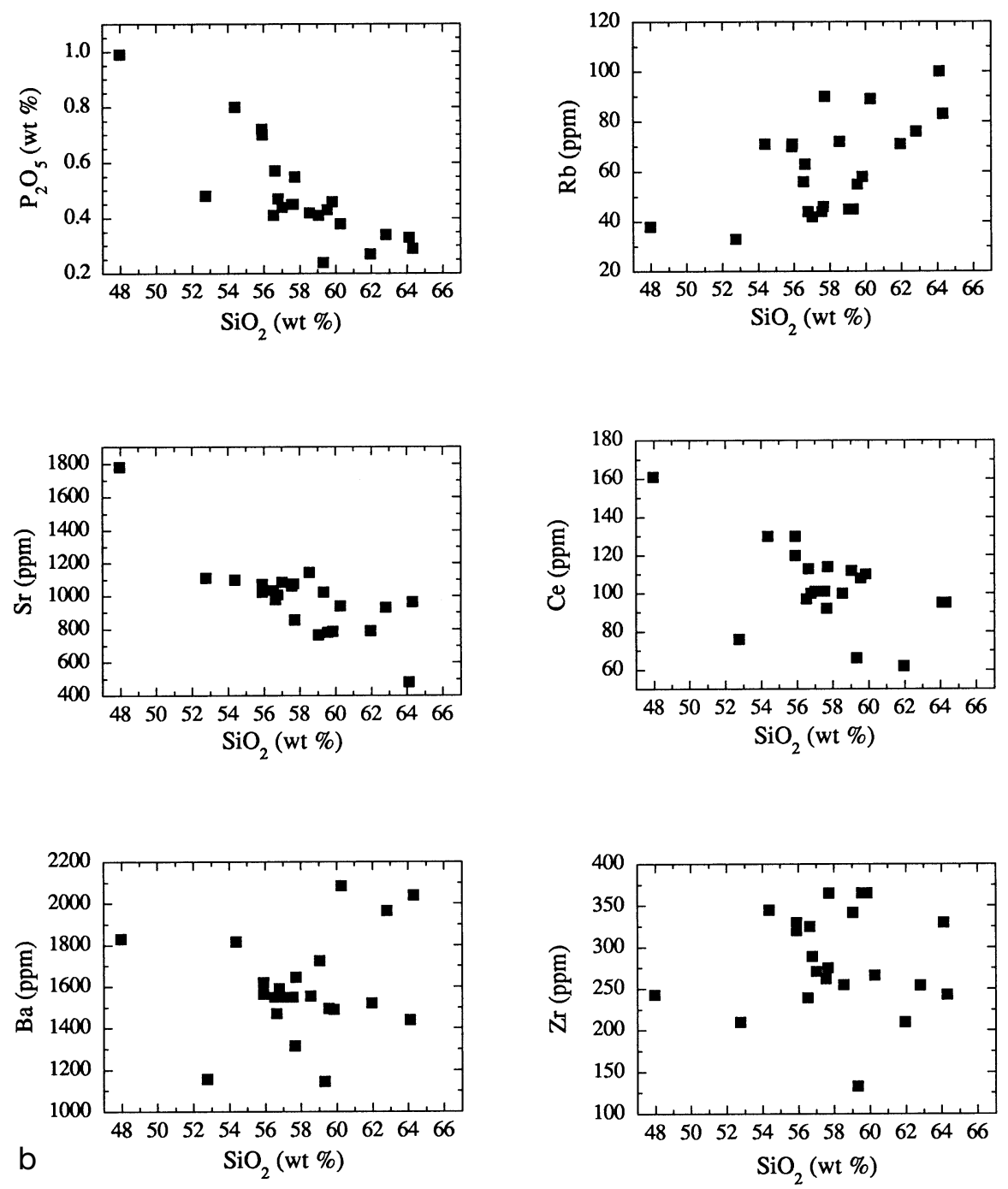

Table 9 Hornblende phenocryst analyses (b.d. below detection limit)

\begin{tabular}{|c|c|c|c|c|c|c|c|c|c|c|c|c|}
\hline & $\begin{array}{l}\text { SN-140 } \\
\text { Core }\end{array}$ & $\begin{array}{l}\text { SN-140 } \\
\text { Rim }\end{array}$ & $\begin{array}{l}\text { SN-25 } \\
\text { Core }\end{array}$ & $\begin{array}{l}\text { SN-25 } \\
\text { Rim }\end{array}$ & $\begin{array}{l}\text { SN-319 } \\
\text { Core }\end{array}$ & $\begin{array}{l}\text { SN-319 } \\
\text { Rim }\end{array}$ & $\begin{array}{l}\text { SN-156 } \\
\text { Core }\end{array}$ & $\begin{array}{l}\text { SN-156 } \\
\text { Rim }\end{array}$ & $\begin{array}{l}\text { SN-31 } \\
\text { Core }\end{array}$ & $\begin{array}{l}\text { SN-31 } \\
\text { Rim }\end{array}$ & $\begin{array}{l}\text { SN-161 } \\
\text { Core }\end{array}$ & $\begin{array}{l}\text { SN-161 } \\
\text { Rim }\end{array}$ \\
\hline $\mathrm{SiO}_{2}$ & 44.8 & 46.3 & 43.9 & 45.3 & 43.3 & 43.0 & 42.0 & 42.8 & 42.6 & 41.8 & 43.6 & 42.9 \\
\hline $\mathrm{TiO}_{2}$ & 1.40 & 1.37 & 2.11 & 2.09 & 3.21 & 3.23 & 2.85 & 2.44 & 3.22 & 3.75 & 2.32 & 2.53 \\
\hline $\mathrm{Al}_{2} \mathrm{O}_{3}$ & 11.8 & 10.5 & 11.0 & 10.1 & 11.8 & 12.0 & 12.8 & 12.1 & 11.5 & 11.7 & 10.4 & 11.0 \\
\hline $\mathrm{Cr}_{2} \mathrm{O}_{3}$ & b.d. & b.d. & b.d. & 0.01 & 0.02 & 0.09 & 0.18 & 0.06 & b.d. & 0.09 & b.d. & b.d. \\
\hline $\mathrm{Fe}_{2} \mathrm{O}_{3}$ & 10.4 & 7.31 & 7.88 & 7.92 & 6.43 & 5.27 & 7.80 & 9.07 & 6.61 & 6.11 & 7.55 & 7.46 \\
\hline $\mathrm{FeO}$ & 0.60 & 1.72 & 4.36 & 4.58 & 6.64 & 7.53 & 6.06 & 5.33 & $5.75^{\mathrm{a}}$ & $5.75^{\mathrm{a}}$ & $5.69^{\mathrm{a}}$ & $5.69^{\mathrm{a}}$ \\
\hline $\mathrm{MnO}$ & 0.19 & 0.14 & 0.15 & 0.24 & 0.13 & 0.19 & 0.18 & 0.23 & 0.19 & 0.13 & 0.27 & 0.20 \\
\hline $\mathrm{MgO}$ & 16.0 & 16.7 & 14.6 & 14.8 & 13.2 & 13.3 & 13.0 & 13.1 & 13.6 & 13.5 & 13.8 & 13.5 \\
\hline $\mathrm{BaO}$ & 0.04 & 0.02 & 0.09 & b.d. & b.d. & 0.09 & 0.15 & 0.08 & b.d. & b.d. & b.d. & b.d. \\
\hline $\mathrm{CaO}$ & 10.4 & 10.9 & 10.9 & 10.8 & 10.5 & 10.9 & 10.7 & 10.5 & 11.5 & 11.8 & 11.4 & 11.5 \\
\hline $\mathrm{Na}_{2} \mathrm{O}$ & 2.59 & 2.31 & 2.18 & 2.03 & 2.22 & 2.41 & 2.46 & 2.27 & 2.14 & 2.26 & 2.09 & 2.12 \\
\hline $\mathrm{K}_{2} \mathrm{O}$ & 0.65 & 0.58 & 0.80 & 0.77 & 0.89 & 0.92 & 0.81 & 0.83 & 1.09 & 1.12 & 0.91 & 0.95 \\
\hline $\mathrm{F}$ & 0.16 & 0.19 & 0.32 & 0.20 & 0.24 & 0.31 & 0.13 & 0.17 & 0.10 & 0.08 & 0.08 & 0.11 \\
\hline $\mathrm{Cl}$ & 0.02 & 0.02 & 0.01 & 0.04 & 0.02 & 0.03 & 0.03 & 0.07 & 0.04 & 0.03 & 0.02 & 0.03 \\
\hline $\mathrm{H}_{2} \mathrm{O}$ & 2.04 & 2.02 & 1.92 & 1.99 & 1.95 & 1.91 & 1.99 & 1.98 & $1.57^{\mathrm{a}}$ & $1.57^{\mathrm{a}}$ & $1.23^{\mathrm{a}}$ & $1.23^{\mathrm{a}}$ \\
\hline $\mathrm{O} \equiv \mathrm{F}$ & -0.07 & -0.08 & -0.14 & -0.08 & -0.10 & -0.13 & -0.05 & -0.07 & -0.04 & -0.03 & -0.03 & -0.05 \\
\hline $\mathrm{O} \equiv \mathrm{Cl}$ & 0.00 & 0.00 & 0.00 & -0.01 & 0.00 & -0.01 & -0.01 & -0.01 & -0.01 & -0.01 & 0.00 & -0.01 \\
\hline Total & 100.8 & 100.1 & 100.2 & 100.7 & 100.4 & 100.8 & 100.6 & 100.9 & 99.7 & 99.6 & 99.3 & 99.1 \\
\hline
\end{tabular}

${ }^{a} \mathrm{FeO}$ by wet chemistry and $\mathrm{H}_{2} \mathrm{O}$ by manometry on pure, mineral separates (free of opaque rims) 
Table 10 Biotite phenocryst analyses (b.d. below detection limit)

\begin{tabular}{lcccccc}
\hline & $\begin{array}{c}\mathrm{SN}-25 \\
\text { Core }\end{array}$ & $\begin{array}{c}\mathrm{SN}-25 \\
\text { Rim }\end{array}$ & $\begin{array}{l}\mathrm{SN}-31 \\
\text { Core }\end{array}$ & $\begin{array}{l}\mathrm{SN}-31 \\
\text { Rim }\end{array}$ & $\begin{array}{l}\mathrm{SN}-161 \\
\text { Core }\end{array}$ & $\begin{array}{l}\mathrm{SN}-161 \\
\text { Rim }\end{array}$ \\
\hline $\mathrm{SiO}_{2}$ & 37.8 & 37.3 & 36.4 & 36.6 & 36.2 & 36.4 \\
$\mathrm{TiO}_{2}$ & 4.40 & 4.37 & 5.20 & 5.18 & 4.75 & 4.66 \\
$\mathrm{Al}_{2} \mathrm{O}_{3}$ & 12.8 & 12.5 & 13.8 & 13.8 & 14.5 & 14.8 \\
$\mathrm{Cr}_{2} \mathrm{O}_{3}$ & b.d. & b.d. & b.d. & b.d. & 0.07 & 0.00 \\
$\mathrm{Fe}_{2} \mathrm{O}_{3}$ & 9.35 & 9.31 & 10.5 & 10.5 & 9.82 & 9.68 \\
$\mathrm{FeO}$ & 7.60 & 7.72 & $7.40^{\mathrm{a}}$ & $7.40^{\mathrm{a}}$ & $4.72^{\mathrm{a}}$ & $4.72^{\mathrm{a}}$ \\
$\mathrm{NiO}$ & 0.02 & 0.03 & b.d. & 0.05 & 0.14 & 0.14 \\
$\mathrm{MnO}$ & 0.19 & 0.14 & 0.22 & 0.22 & 0.10 & 0.14 \\
$\mathrm{MgO}$ & 15.0 & 14.7 & 12.3 & 13.0 & 15.8 & 16.0 \\
$\mathrm{BaO}$ & 1.24 & 1.02 & 1.50 & 1.11 & 1.43 & 1.06 \\
$\mathrm{CaO}$ & 0.01 & 0.02 & 0.02 & 0.05 & 0.06 & 0.03 \\
$\mathrm{Na}{ }_{2} \mathrm{O}$ & 0.59 & 0.71 & 0.59 & 0.62 & 0.91 & 0.79 \\
$\mathrm{~K} \mathrm{O}$ & 8.65 & 8.58 & 9.09 & 9.02 & 8.37 & 8.76 \\
$\mathrm{~F}$ & 0.06 & 0.09 & 0.15 & 0.09 & 0.03 & 0.09 \\
$\mathrm{Cl}$ & 0.02 & 0.02 & 0.06 & 0.06 & 0.05 & 0.06 \\
$\mathrm{H}_{2} \mathrm{O}$ & 2.04 & 2.02 & $1.57^{\mathrm{a}}$ & $1.57^{\mathrm{a}}$ & $2.41^{\mathrm{a}}$ & $2.41^{\mathrm{a}}$ \\
$\mathrm{O} \equiv \mathrm{F}$ & -0.07 & -0.08 & -0.06 & -0.04 & -0.01 & -0.04 \\
$\mathrm{O} \equiv \mathrm{Cl}$ & 0.00 & 0.00 & -0.01 & -0.01 & -0.01 & -0.01 \\
$\mathrm{Total}$ & 99.6 & 98.5 & 98.7 & 99.1 & 99.3 & 99.7 \\
\end{tabular}

${ }^{a} \mathrm{FeO}$ by wet chemistry and $\mathrm{H}_{2} \mathrm{O}$ by manometery on pure, mineral separates (free of opaque rims)

Table 11 Representative analyses of Cr-spinel inclusions

\begin{tabular}{|c|c|c|c|c|c|}
\hline & $\mathrm{SN}-23$ & SN-347 & SN-303 & SN-311 & SN-160 \\
\hline $\mathrm{SiO}_{2}$ & 0.11 & 0.24 & 0.07 & 0.26 & 0.14 \\
\hline $\mathrm{TiO}_{2}$ & 0.64 & 3.60 & 7.29 & 6.65 & 11.4 \\
\hline $\mathrm{Al}_{2} \mathrm{O}_{3}$ & 33.8 & 20.0 & 9.58 & 3.02 & 4.33 \\
\hline $\mathrm{V}_{2} \mathrm{O}_{3}$ & 0.18 & 0.38 & 0.25 & 0.47 & 0.69 \\
\hline $\mathrm{Cr}_{2} \mathrm{O}_{3}$ & 26.5 & 19.3 & 11.3 & 4.21 & 15.3 \\
\hline $\mathrm{FeO}$ & 21.0 & 44.2 & 60.6 & 77.8 & 60.5 \\
\hline $\mathrm{MnO}$ & 0.11 & 0.48 & 0.31 & 0.50 & 0.72 \\
\hline $\mathrm{MgO}$ & 16.6 & 9.10 & 6.47 & 1.87 & 4.38 \\
\hline $\mathrm{CaO}$ & b.d. & 0.15 & b.d. & 0.19 & 0.08 \\
\hline $\mathrm{NiO}$ & 0.16 & 0.49 & 0.11 & 0.53 & 0.57 \\
\hline Total & 99.0 & 98.0 & 96.0 & 95.5 & 98.3 \\
\hline $\mathrm{Fe}_{2} \mathrm{O}_{3}$ & 9.58 & 23.1 & 34.9 & 48.5 & 29.3 \\
\hline $\mathrm{FeO}$ & 12.4 & 23.5 & 29.2 & 34.1 & 35.1 \\
\hline Total & 100.0 & 100.3 & 99.5 & 100.3 & 100.9 \\
\hline $\mathrm{Cr} \#$ & 0.34 & 0.39 & 0.44 & 0.48 & 0.70 \\
\hline $\mathrm{Mg} \#$ & 0.71 & 0.41 & 0.28 & 0.09 & 0.18 \\
\hline $\mathrm{Fe}^{3+} \#$ & 0.11 & 0.31 & 0.57 & 0.84 & 0.55 \\
\hline
\end{tabular}

\section{Water content}

The diversity of phenocryst assemblages observed in the andesite lavas of the Aurora volcanic field indicates that pre-eruptive water contents were both high and variable. The presence of hydrous phases such as hornblende and biotite in many of the lavas (Table 2) attests to water contents in excess of $2 \mathrm{wt} \% \mathrm{H}_{2} \mathrm{O}$ (Baker and Eggler 1987; Sisson and Grove 1993). Moreover, the absence of plagioclase phenocrysts in the Aurora Crater two-pyroxene andesite lavas, with $>59 \mathrm{wt} \% \mathrm{SiO}_{2}$, further indicates the role of water in suppressing the early crystallization of plagioclase. The hornblende lamprophyre (sample 140) with $\sim 60 \mathrm{wt} \% \mathrm{SiO}_{2}$ is remarkable for its lack of plagioclase phenocrysts, despite crystallization of $\sim 14 \mathrm{vol} \%$ hornblende. The phase equilibrium experiments of Luhr (1990) and Moore (1995) on liquids of similar bulk composition to sample 140 indicate that $\sim 6 \mathrm{wt} \% \mathrm{H}_{2} \mathrm{O}$ is required to reproduce this phenocryst assemblage.

Broad constraints on the water contents in the plagioclase-bearing Aurora magmas can be made using the exchange of $\mathrm{Ca}$ and $\mathrm{Na}$ between plagioclase and liquid $\left(\mathrm{K}_{\mathrm{D}}{ }^{\mathrm{Ca}-\mathrm{Na}}\right)$, which is strongly dependent on liquid composition and water contents (Tsuchiyama 1985; Baker and Eggler 1987; Rutherford and Devine 1988; Luhr 1990; Sisson and Grove 1993) and increases in water-saturated andesite and high-alumina basalt from $\sim 1.7$ to $\sim 5.5$ at 2 to $6 \mathrm{wt} \% \mathrm{H}_{2} \mathrm{O}$ respectively. In Fig. 6 , values of $\mathrm{Ca} /$ $(\mathrm{Ca}+\mathrm{Na})$ for the groundmass compositions in Table 13 are plotted against the range of plagioclase rim compositions found for each sample. Isopleths of constant $\mathrm{K}_{\mathrm{D}}{ }^{\mathrm{Ca}-\mathrm{Na}}$ equal to 2, 3, 4 and 5 (equivalent to $\sim 2.4,3.4$, 4.5 and $5.5 \mathrm{wt} \% \mathrm{H}_{2} \mathrm{O}$ in the melt, respectively) are superimposed on these data. All of the hornblende andesites have plagioclase rim compositions that fall within a fairly narrow interval $(\sim 10 \mathrm{~mol} \% \mathrm{An})$ and plot above the $\mathrm{K}_{\mathrm{D}}{ }^{\mathrm{Ca}-\mathrm{Na}}$ isopleth of 3 (corresponding to a melt water content of $\sim 3.4 \mathrm{wt} \% \mathrm{H}_{2} \mathrm{O}$ ). Sample 161, which contains the most abundant biotite (Table 1), may have had as much as $6 \mathrm{wt} \% \mathrm{H}_{2} \mathrm{O}$ in the melt during growth of its plagioclase. In contrast, sample 26 , which contains no hydrous phenocryst phase appears to have had less than $2 \mathrm{wt} \% \mathrm{H}_{2} \mathrm{O}$ in its melt prior to eruption.

\section{Oxygen fugacity}

Unfortunately, ilmenite does not occur with titanomagnetite in any of the lavas from the Aurora volcanic field, thus preventing utilization of two Fe-Ti oxides to determine oxygen fugacity. Oxygen fugacity can be estimated, however, by a more general method using whole-rock $\mathrm{Fe}_{2} \mathrm{O}_{3} / \mathrm{FeO}$ values and the experimentally calibrated relationship involving temperature, oxygen fugacity and bulk composition (e.g., Kress and Carmichael 1991). Moore et al. (1995) have shown that this model can be applied to hydrous silicate liquids, since dissolved water has no measurable effect on the oxidation state of iron in a silicate melt. Analytical errors on ferric-ferrous determinations, and on the calibration of Kress and Carmichael (1991), lead to an uncertainty in $f_{\mathrm{O}_{2}}$ of \pm 0.7 $\log$ units $(2 \sigma)$. However, these estimates of oxygen fugacity, relative to the $\mathrm{Ni}-\mathrm{NiO}$ buffer $\left(\Delta \mathrm{NNO}=\log f_{\mathrm{O}_{2}}\right.$ (magma) $-\log f_{\mathrm{O}_{2}}(\mathrm{Ni}-\mathrm{NiO}$ buffer $)$, must be made with caution since post-eruptive alteration can alter bulk ferric-ferrous ratios. This is a particular concern in the Aurora volcanic field because of its partial burial beneath an ancestral Mono Lake.

An excellent way to test whether the bulk ferric-ferrous ratio of a lava has been altered is to compare the composition of the phenocryst cores of either olivine or 
Table 12 Representative groundmass Fe-Ti oxide analyses (b.d. below detection limit)

SN-23 SN-347 SN-303 SN-368 SN-26 SN-311 SN-160 SN-156 SN-162 SN-324 SN-326 SN-140 SN-25 SN-319 SN-31 SN-161

\begin{tabular}{|c|c|c|c|c|c|c|c|c|c|c|c|c|c|c|c|c|}
\hline $\mathrm{SiO}_{2}$ & 0.42 & 0.26 & 0.16 & 0.09 & 0.09 & 0.24 & 0.24 & 0.12 & 0.14 & 0.07 & 0.07 & 0.13 & 0.07 & 0.07 & 0.15 & 0.10 \\
\hline $\mathrm{O}_{2}$ & 9.80 & 19.1 & 16.4 & 9.06 & 14.6 & 12.7 & 14.9 & 8.8 & 13.2 & 16.3 & 16.1 & 10.7 & 6.28 & 8.85 & 6.25 & 6.34 \\
\hline $\mathrm{l}_{2} \mathrm{O}_{3}$ & 5.28 & 1.35 & 2.32 & 1.09 & 3.13 & 2.24 & 1.43 & 3.45 & 2.58 & 2.85 & 2.53 & 1.17 & 1.95 & 4.29 & 3.34 & 2.82 \\
\hline $\mathrm{V}_{2} \mathrm{O}_{3}$ & 0.42 & 0.74 & 0.43 & 0.46 & 0.83 & 0.63 & 0.88 & 0.40 & 0.59 & 0.89 & 0.68 & 0.49 & 0.21 & 0.53 & 0.35 & 0.21 \\
\hline $\mathrm{Cr}_{2} \mathrm{O}_{3}$ & 1.88 & 2.25 & 0.91 & 0.26 & 1.45 & 0.58 & 1.05 & 0.06 & 0.26 & 0.70 & 0.20 & 0.01 & 0.04 & b.d. & 0.12 & 0.13 \\
\hline $\mathrm{O}$ & 68.9 & 68.1 & 70.9 & 81.5 & 73.2 & 74.6 & 76.2 & 79.1 & 76.1 & 73.2 & 72.0 & 80.4 & 82.9 & 77.9 & 82.0 & 83.5 \\
\hline $\mathrm{nO}$ & 0.85 & 0.85 & 0.73 & 0.35 & 0.35 & 0.92 & 0.53 & 0.38 & 0.46 & 0.89 & 0.49 & 0.41 & 0.82 & 0.33 & 0.43 & 0.48 \\
\hline $\mathrm{gO}$ & 6 & 6 & 2.9 & 1.30 & 2.66 & $3 .($ & 0.99 & 2. & 2. & 1.60 & 3. & & 1.2 & & 9 & 1.29 \\
\hline $\mathrm{OO}$ & 1.2 & 0.44 & 0.17 & 0.15 & 0.03 & 0.2 & 0.21 & 0.04 & 0.09 & 0.32 & 0.01 & 0.11 & 0.04 & b.c & 0.14 & 0.03 \\
\hline $\mathrm{iO}$ & 0.52 & 0.51 & b.d. & b.d. & 0.01 & 0.42 & 0.53 & b.d. & b.d & 0.47 & 0.04 & b.d. & b.d. & b.d. & 0.05 & 0.00 \\
\hline tal & 95.3 & 97.1 & 95.0 & .2 & 96.3 & 9 & 9 & לד. & 95.4 & .5 & . & , & 93.6 & 94.5 & 042 & . \\
\hline${ }_{2} \mathrm{O}_{3}$ & 43.9 & 28.8 & 33.4 & 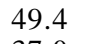 & 36.0 & 41. & 7.0 & 48 & 40. & 33.2 & 35. & 46 & 54 & 47. & 52. & 53.7 \\
\hline $\mathrm{O}$ & 29.4 & 42.2 & 40.9 & 37.0 & 40.8 & 37.2 & 42.9 & 35.7 & 40.1 & 43.3 & 40.4 & 38.4 & 34.2 & 35.6 & 34.7 & 35.1 \\
\hline Total & 99.7 & 100.0 & 98.4 & 99.2 & 99.9 & 99.8 & 100.6 & 99.7 & 99.5 & 100.5 & 99.1 & 99.6 & 99.1 & 99.2 & 99.4 & 100.2 \\
\hline$X_{\text {Mte }}$ & 0 & 0 & 0. & 0 & 0.5 & 0. & 0.52 & 0. & 0.5 & 0.46 & 0.4 & 0. & 0. & 0. & 0 . & 0.75 \\
\hline$X_{\mathrm{Ulv}}$ & 0.28 & 0.53 & 0.46 & 0.26 & 0.41 & 0.36 & 0.42 & 0.25 & 0.37 & 0.46 & 0.45 & 0.31 & 0.18 & 0.25 & 0.18 & 0.18 \\
\hline
\end{tabular}

Table 13 Electron microprobe analyses of groundmass $(m c$ microcrystalline, $g l$ glass)

\begin{tabular}{lccccccccc}
\hline & $\begin{array}{l}\mathrm{SN}-368 \\
\mathrm{mc}\end{array}$ & $\begin{array}{l}\mathrm{SN}-26 \\
\mathrm{mc}\end{array}$ & $\begin{array}{l}\mathrm{SN}-328 \\
\mathrm{mc}\end{array}$ & $\begin{array}{l}\mathrm{SN}-313 \\
\mathrm{mc}\end{array}$ & $\begin{array}{l}\mathrm{SN}-319 \\
\mathrm{mc}\end{array}$ & $\begin{array}{l}\mathrm{SN}-326 \\
\mathrm{gl}\end{array}$ & $\begin{array}{l}\mathrm{SN}-25 \\
\mathrm{mc}\end{array}$ & $\begin{array}{l}\mathrm{SN}-31 \\
\mathrm{mc}\end{array}$ & $\begin{array}{l}\mathrm{SN}-161 \\
\mathrm{mc}\end{array}$ \\
\hline $\mathrm{SiO}_{2}$ & 59.7 & 60.8 & 61.3 & 61.6 & 62.1 & 66.5 & 67.7 & 68.8 & 71.0 \\
$\mathrm{TiO}_{2}$ & 0.94 & 1.44 & 0.67 & 0.69 & 0.70 & 0.99 & 0.29 & 0.45 & 0.41 \\
$\mathrm{Al}_{2} \mathrm{O}_{3}$ & 19.4 & 15.2 & 17.8 & 18.7 & 17.6 & 15.3 & 16.6 & 15.3 & 14.4 \\
$\mathrm{FeO}^{\mathrm{T}}$ & 4.09 & 5.32 & 4.12 & 2.23 & 4.25 & 3.23 & 1.73 & 1.80 & 1.68 \\
$\mathrm{MnO}$ & 0.20 & 0.24 & 0.16 & 0.17 & 0.21 & 0.03 & 0.01 & 0.12 & 0.19 \\
$\mathrm{MgO}$ & 1.04 & 1.54 & 2.07 & 0.81 & 1.66 & 0.57 & 0.52 & 0.52 & 0.49 \\
$\mathrm{CaO}$ & 3.99 & 3.55 & 3.75 & 3.21 & 3.47 & 1.51 & 2.48 & 1.67 & 0.86 \\
$\mathrm{Na} 2$ & 5.08 & 3.84 & 4.59 & 4.90 & 4.44 & 4.23 & 4.37 & 4.21 & 3.98 \\
$\mathrm{~K}_{2} \mathrm{O}$ & 4.72 & 5.62 & 4.51 & 4.65 & 3.98 & 6.28 & 4.39 & 5.11 & 5.31 \\
$\mathrm{P}_{2} \mathrm{O}_{5}$ & 0.48 & 0.83 & 0.12 & 0.38 & 0.44 & 0.19 & 0.09 & 0.17 & 0.21 \\
$\mathrm{Total}$ & 99.6 & 98.4 & 99.1 & 97.3 & 98.8 & 98.8 & 98.2 & 98.1 & 98.5 \\
\hline
\end{tabular}

orthopyroxene to that predicted on the basis of the experimentally based FeO-MgO exchange between phenocryst and liquid. Moore (1995) have developed a revised empirical expression for this equilibrium exchange based on a data set of 299 experimental olivine-natural liquid pairs. The most magnesian core composition that is observed in each sample is assumed to represent the composition of olivine that first began to crystallize at the liquidus; this core composition can then be used, in conjuction with the calculated $K_{D}$ value, to infer the ferric-ferrous ratio and hence $f_{\mathrm{O}_{2}}$ of the bulk liquid prior to eruption.

The estimates of $f_{\mathrm{O}_{2}}$ obtained in this manner can be compared to those derived from the whole-rock ferricferrous ratios; deviations range between 1.1 and $0.2 \mathrm{log}$ units. In contrast to all other samples, the olivine in sample 160 is much too fayalitic to have been in equilibrium with the host liquid under any $f_{\mathrm{O}_{2}}$ condition and is undoubtedly xenocrystic. The results of this test indicate that when there is obvious petrographic evidence for post-eruptive oxidation (e.g., iddingsite rims on olivine), bulk ferric-ferrous ratios may give $\triangle \mathrm{NNO}$ values that are more than one log unit too high. The highest $\Delta$ NNO value 


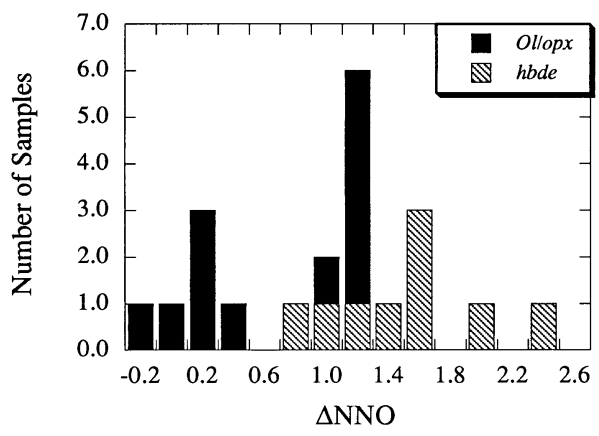

Fig. 7 Histogram of $\triangle \mathrm{NNO}: \log f_{\mathrm{O}_{2}}$ (sample) $-\log f_{\mathrm{O}_{2}}(\mathrm{Ni}-\mathrm{NiO}$ buffer) for lavas from the Aurora volcanic field. Solid pattern denotes samples with olivine \pm orthopyroxene phenocrysts, whereas hatched pattern denotes samples with hornblende \pm biotite phenocrysts

$(+2.4)$ for a lava that is demonstrably pristine and was never covered by the ancestral Mono Lake is from Aurora Peak (sample 161), a biotite-hornblende andesite. A histogram of $\Delta \mathrm{NNO}$ values is presented in Fig. 7. The important conclusion is that none of the Aurora magmas had pre-eruptive oxygen fugacities lower than the Ni$\mathrm{NiO}$ buffer. This result is consistent with the absence of ilmenite and pigeonite in all samples, both of which are favored at $f_{\mathrm{O}_{2}}$ values $\leq$ QFM (Grove and Juster 1989; Juster et al. 1989; Snyder et al. 1993; Toplis and Carroll 1995).

\section{Differentiation trends}

The Aurora magmas record a classic calc-alkaline differentiation trend from basalt through dacite. The generation of the Mt Hicks high-silica rhyolite is treated in another study. In this discussion we explore three different mechanisms that are commonly invoked to explain a basalt through dacite continuum: (1) partial melting of granitoid, followed by mixing and assimilation with basalt, (2) crystal fractionation in largely molten crustal magma chambers, and (3) re-working of mantle-derived melts at the base of the crust. The mineralogy and composition of the magmas, their intensive variables, and their eruption rates are all used to evaluate the relative importance of these three processes during differentiation of the Aurora suite.

Partial melting of granitoid basement, magma mixing and assimilation

The first model to test is partial melting of granitoid basement, followed by mixing with mantle-derived basalt to generate andesitic magmas, a process that has been well documented at the Medicine Lake volcano in northeastern California (Grove et al. 1988). Although the Cretaceous plutons in the Mono Basin region have a distinctive and narrow range of ${ }^{87} \mathrm{Sr} /{ }^{86} \mathrm{Sr}$ values $(0.7066-$
0.7071; Kistler and Peterman 1978), Sr isotopes cannot be used to resolve their involvement in the Aurora magmas. All of the mafic, potassic lavas in the Mono BasinLong Valley area have elevated ${ }^{87} \mathrm{Sr} /{ }^{86} \mathrm{Sr}$ values (0.70604-0.70652; Nielsen et al. 1991) that overlap those measured for ultrapotassic Pliocene volcanic rocks erupted in the central Sierra Nevada (0.70597-0.70653; Van Kooten 1981). Moreover, some of the Sierran potassic lavas contain pyroxenite and peridotite mantle xenoliths with ${ }^{87} \mathrm{Sr} /{ }^{86} \mathrm{Sr}$ values of $0.70448-0.70687$ (Van Kooten et al. 1985), indicating that crustal contamination of the Sierran granitoids is not required to explain the elevated $\mathrm{Sr}$ isotopic values of the potassic magmas. This does not mean, however, that it did not occur.

The presence of sparse, quartz xenocrysts in some Aurora flows attests to some incorporation of crustal material, probably from the granitoid basement. It is not clear, however, whether the quartz xenocrysts represent small, solid fragments of crust that were incorporated but not fully assimilated during transport of the magma to the surface, or alternatively, reflect significant granitoid melting, mixing, and assimilation.

Mixing of basalt with rhyolite or dacite (partial melts of granitoid) to produce intermediate magmas (basaltic andesite and andesite) has been well-documented in numerous volcanic systems, including the Coso volcanic field (Bacon and Metz 1984), Medicine Lake volcano (Grove et al. 1988), and Mt. Pinatubo (Pallister et al. 1992). In each case, the mixed magmas display unambiguous textural evidence of their hybrid origin. At the Coso volcanic field, blob-like inclusions of a hybrid andesite, formed at the interface between a basaltic and a rhyolitic magma in a zoned chamber, are found in several rhyolite domes. No large phenocrysts grew from the mixed magma, but resorbed and relict phenocrysts of both basaltic and rhyoltic origin coexist in the andesite inclusions (Bacon and Metz 1984). At Medicine Lake volcano, the Burnt Lava andesite flow contains abundant inclusions of both highly melted granitic crustal xenoliths (1-6 cm in size) and high-Al basalt ( $\mathrm{mm}$ to $\mathrm{cm}$ scale); the xenocrysts and phenocrysts in the Burnt Lava flow are frequently reacted, resorbed and overgrown to display reversed zoning (Grove et al. 1988). Finally, the well-documented 1991 eruption of Mt. Pinatubo produced a mixed andesite (in the initial dome and tephra emissions) that is a heterogeneous mixture of approximately 60:40 dacite and basalt, with $\mathrm{mm}$ to $\mathrm{cm}$ sized blobs of basalt within the dacite (Pallister et al. 1992).

In sharp contrast to these examples, the vast majority of the Aurora lavas display no such textures. Normal zoning is found for all olivine phenocrysts in basalt, basaltic andesite and andesite samples (although the normally zoned, Fe-rich olivine in sample 160 must be xenocrystic). The biotite-hornblende andesites (samples 25, 31 and 161) all have clear, euhedral phenocrysts of plagioclase, hornblende and biotite with normal zoning. Moreover, the two-pyroxene andesites of the Aurora Crater (samples 162, 328 and 329) and the Cedar Hill complex (326), as well as the thin, capping hornblende andesite 
flow (sample 319) also display normal zoning of their phenocrysts, with no "second" population of phenocrysts or xenocrysts. In fact, most of the Aurora andesites and basaltic andesites are remarkable for the pristine and primary character of their phenocrysts. The only flows with evidence for disequilibria are from cone B (sample 368; Fig. 1) and the youngest lava flow (sample 156; Fig. 1); both samples contain plagioclase with sieve-like reaction rims, and sample 156 contains reversely zoned augite phenocrysts. However, these two flows, comprising $<10 \%$ of the erupted volume of the Aurora volcanic field, are the exception rather than the rule.

Complete mixing between a mantle-derived basalt and a siliceous, partial melt of granitoid to form an andesite liquid can occur on a geologically reasonable time scale only if the length scale for chemical diffusion is sufficiently small. This would require turbulent mixing of rhyolitic and basaltic magma, which although disfavored by the large viscosity contrasts between the two end-members, could possibly occur on a local scale at an interface to produce small volumes of mixed magmas (e.g., the Mt. Pinatubo andesite; Snyder and Tait 1996). It is doubtful, however, that such mixed magmas would not record their hybrid nature in their phenocryst assemblage, since the temperature contrast between a siliceous, crustal melt and a basalt would promote crystallization and tend to fossilize the mixed magma prior to complete homogenization. Indeed, there is abundant field evidence for the quenching of basaltic magmas into pillows after their injection into silicic magma chambers (e.g., Frost and Mahood 1987; Michael 1991; Wiebe 1993). Although there is no doubt that small volumes of hybrid, intermediate magma can be formed by turbulent mixing of basalt and rhyolite, it is unlikely that this process can account for the $>15 \mathrm{~km}^{3}$ of intermediate magma that erupted in the Aurora volcanic field, in which no textural or phenocryst evidence of mixing is recorded.

\section{Crystal fractionation}

The second mechanism for differentiation that can be tested is that of fractionational crystallization involving phenocryst minerals. This process is explored in Fig. 8, in which the bulk versus groundmass composition (Table 13) of several Aurora lavas are compared on a plot of $\mathrm{FeO}^{\mathrm{T}} / \mathrm{MgO}$ vs $\mathrm{SiO}_{2}$. For many samples, separation of the phenocryst assemblage (that does not include titanomagnetite) leads to an iron enrichment in the residual liquid that is counter to the general calc-alkaline trend of the Aurora suite as a whole. Thus it does not appear that separation of observed phenocrysts through a crystal fractionation process can explain the differentiation of the Aurora magmas. Alternatively, crystal fractionation may have occurred at a greater depth than that at which the phenocrysts grew, and with a different assemblage and/or proportions of crystals from that observed in the erupted lavas.

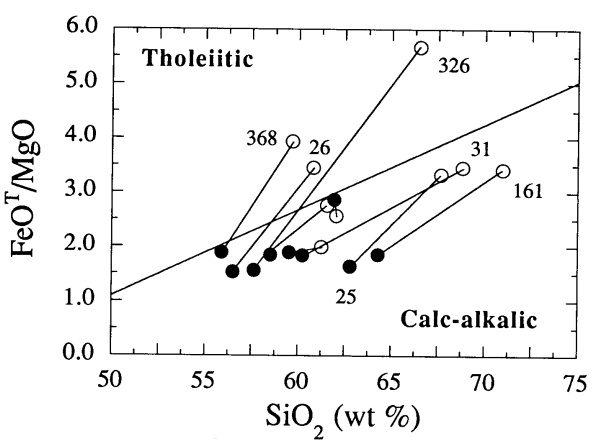

Fig. 8 A plot of $\mathrm{FeO}^{\mathrm{T}} / \mathrm{MgO}$ vs $\mathrm{SiO}_{2}$ for several samples for which both the bulk composition (solid squares) and the groundmass composition (open squares) are known. Separation of the phenocryst assemblage frequently leads to an iron enrichment in the residual liquid that is counter to the general calc-alkaline trend of the Aurora suite

Gill (1981) strongly favored crystal fractionation of the assemblage plagioclase, olivine, augite and magnetite (POAM) as the dominant mechanism for the derivation of andesite from basalt along a calc-alkaline trend; removal of titanomagnetite is required to suppress the enrichment of iron that is so characteristic of tholeiitic magmas, while at the same time increasing the silica content of the residual liquids. The linear trends of decreasing $\mathrm{TiO}_{2}$ and $\mathrm{FeO}^{\mathrm{T}}$ contents with $\mathrm{SiO}_{2}$ concentration in the Aurora magmas (Fig. 5a) are consistent with early and continuous participation of titanomagnetite in the fractionating assemblage. Such a role for titanomagnetite is plausible given the high water and oxygen fugacities inferred for these magmas, which together put titanomagnetite close to the liquidus (Sisson and Grove 1993).

In the following discussion, we explore the effect of crystal fractionation on the relative oxygen fugacity of residual liquids. Once a melt has segregated from its source region, its $f_{\mathrm{O}_{2}}$ is no longer buffered, but is uniquely defined by its bulk composition, ferric-ferrous ratio, temperature and pressure. During fractional crystallization, as different mineral phases are removed with ferricferrous ratios that are different from that of the host liquid, the ferric-ferrous ratio and hence relative $f_{\mathrm{O}_{2}}$ (relative to the Ni-NiO buffer to correct for the effect of changing temperature) of the residual melt will change, as demonstrated below.

Fractionation calculations were made using the program XLFRAC of Stormer and Nicholls (1978); the program was modified to include $\mathrm{Fe}_{2} \mathrm{O}_{3}$ as a component. The results of all fractionation models are reported in Table 14. Following Grove and Kinzler (1986), fractionation from an initial basalt composition (sample 23) to a final andesite composition (sample 162) was divided into two stages, with an intermediate basaltic andesite composition (sample 303). Thus the net proportion and composition of minerals in the fractionating assemblage were allowed to change between the two stages. The ferricferrous ratios in the parental liquids (samples 23 and 
Table 14a XLFRAC calculations of olivine, plagioclase, clinopyroxene and magnetite

\begin{tabular}{|c|c|c|c|c|c|c|c|c|c|c|}
\hline \multirow[t]{2}{*}{ Liquids } & \multicolumn{9}{|c|}{$\mathrm{Wt} \%$ oxides } & \multirow[t]{2}{*}{$\triangle \mathrm{NNO}$} \\
\hline & $\mathrm{SiO}_{2}$ & $\mathrm{TiO}_{2}$ & $\mathrm{Al}_{2} \mathrm{O}_{3}$ & $\mathrm{Fe}_{2} \mathrm{O}_{3}$ & $\mathrm{FeO}$ & $\mathrm{MgO}$ & $\mathrm{CaO}$ & $\mathrm{Na}_{2} \mathrm{O}$ & $\mathrm{K}_{2} \mathrm{O}$ & \\
\hline \multicolumn{11}{|l|}{ Stage 1} \\
\hline $\begin{array}{l}\text { Initial composition (SN-23) } \\
\text { Intermediate composition (SN-303) } \\
\text { Calculated composition (SN-303*) }\end{array}$ & $\begin{array}{l}49.4 \\
55.8 \\
55.3\end{array}$ & $\begin{array}{l}1.53 \\
1.38 \\
1.59\end{array}$ & $\begin{array}{l}16.8 \\
17.9 \\
17.8\end{array}$ & $\begin{array}{l}1.81 \\
1.60 \\
0.85\end{array}$ & $\begin{array}{l}6.21 \\
5.38 \\
5.34\end{array}$ & $\begin{array}{l}7.91 \\
3.74 \\
3.60\end{array}$ & $\begin{array}{c}10.4 \\
6.10 \\
6.27\end{array}$ & $\begin{array}{l}3.15 \\
4.38 \\
4.55\end{array}$ & $\begin{array}{l}2.65 \\
3.55 \\
4.65\end{array}$ & $\begin{array}{r}0.0 \\
0.0 \\
-1.6\end{array}$ \\
\hline \multicolumn{11}{|l|}{ Stage $2 \mathrm{a}$} \\
\hline $\begin{array}{l}\text { Initial composition }(\mathrm{SN}-303 *) \\
\text { Evolved composition }(\mathrm{SN}-162) \\
\text { Calculated composition }(\mathrm{SN}-162 *)\end{array}$ & $\begin{array}{l}55.9 \\
60.5 \\
60.1\end{array}$ & $\begin{array}{l}1.38 \\
0.95 \\
1.31\end{array}$ & $\begin{array}{l}17.9 \\
17.1 \\
16.9\end{array}$ & $\begin{array}{l}0.87 \\
1.28 \\
0.03\end{array}$ & $\begin{array}{l}5.99 \\
4.24 \\
4.76\end{array}$ & $\begin{array}{l}3.74 \\
2.93 \\
2.37\end{array}$ & $\begin{array}{l}6.11 \\
5.23 \\
5.51\end{array}$ & $\begin{array}{l}4.38 \\
4.14 \\
4.55\end{array}$ & $\begin{array}{l}3.56 \\
3.47 \\
4.36\end{array}$ & $\begin{array}{r}-1.6 \\
0.0 \\
-8.8\end{array}$ \\
\hline \multicolumn{11}{|l|}{ Stage $2 b$} \\
\hline $\begin{array}{l}\text { Initial composition (SN-303) } \\
\text { Evolved composition (SN-162) } \\
\text { Calculated composition }(\mathrm{SN}-162 * *)\end{array}$ & $\begin{array}{l}55.8 \\
60.5 \\
60.1\end{array}$ & $\begin{array}{l}1.38 \\
0.95 \\
1.24\end{array}$ & $\begin{array}{l}17.9 \\
17.1 \\
16.9\end{array}$ & $\begin{array}{l}1.60 \\
1.28 \\
0.56\end{array}$ & $\begin{array}{l}5.38 \\
4.24 \\
4.20\end{array}$ & $\begin{array}{l}3.74 \\
2.93 \\
2.68\end{array}$ & $\begin{array}{l}6.10 \\
5.23 \\
5.32\end{array}$ & $\begin{array}{l}4.38 \\
4.14 \\
4.56\end{array}$ & $\begin{array}{l}3.55 \\
3.47 \\
4.36\end{array}$ & $\begin{array}{r}0.0 \\
0.0 \\
-2.0\end{array}$ \\
\hline
\end{tabular}

Table 14b Fractionation calculations for Aurora lavas

\begin{tabular}{|c|c|c|c|c|}
\hline & $\begin{array}{l}\text { Olivine } \\
\text { (Fo) }\end{array}$ & $\begin{array}{l}\text { Augite } \\
(\text { En/Fs })\end{array}$ & $\begin{array}{l}\text { Plagioclase } \\
\text { (An) }\end{array}$ & $\begin{array}{l}\text { Titanomagnetite } \\
\text { (Mag) }\end{array}$ \\
\hline \multicolumn{5}{|l|}{ Stage 1} \\
\hline $\begin{array}{l}\text { Compositions predicted to be in equilibrium } \\
\text { with range of liquids } \\
\text { Proportions removed in calculation }\end{array}$ & $\begin{array}{l}0.87-0.79 \\
-8.0 \%\end{array}$ & $\begin{array}{l}0.42 / 0.11 \\
-19.4 \%\end{array}$ & $\begin{array}{l}0.70-0.60 \\
-28.7 \%\end{array}$ & $\begin{aligned} & 0.48\left(\mathrm{Fe}^{3+} / \Sigma \mathrm{Fe}=0.44\right) \\
- & 3.8 \%\end{aligned}$ \\
\hline \multicolumn{5}{|l|}{ Stage $2 \mathrm{a}$} \\
\hline $\begin{array}{l}\text { Compositions predicted to be in equilibrium } \\
\text { with range of liquids } \\
\text { Proportions removed in calculation }\end{array}$ & $\begin{array}{l}0.79-0.69 \\
-5.9 \%\end{array}$ & $\begin{array}{l}0.40 / 0.15 \\
+0.8 \%\end{array}$ & $\begin{array}{l}0.67-0.41 \\
-18.9 \%\end{array}$ & $\begin{array}{l}0.56\left(\mathrm{Fe}^{3+} / \Sigma \mathrm{Fe}=0.47\right) \\
-2.5 \%\end{array}$ \\
\hline \multicolumn{5}{|l|}{ Stage $2 b$} \\
\hline $\begin{array}{l}\text { Compositions predicted to be in equilibrium } \\
\text { with range of liquids } \\
\text { Proportions removed in calculation }\end{array}$ & $\begin{array}{l}0.79-0.69 \\
-4.6 \%\end{array}$ & $\begin{array}{l}0.40 / 0.15 \\
-0.3 \%\end{array}$ & $\begin{array}{l}0.67-0.41 \\
-18.9 \%\end{array}$ & $\begin{aligned} & 0.56\left(\mathrm{Fe}^{3+} / \Sigma \mathrm{Fe}=0.47\right) \\
- & 2.9 \%\end{aligned}$ \\
\hline
\end{tabular}

$303)$ are those calculated for the reported $\triangle \mathrm{NNO}$ values at starting temperatures of 1200 and $1100^{\circ} \mathrm{C}$, respectively.

The first stage involves crystal fractionation of the average observed phenocryst compositions: olivine $\left(\mathrm{Fo}_{83}\right)$, clinopyroxene $\left(\mathrm{En}_{44} / \mathrm{Fs}_{11}\right)$, plagioclase $\left(\mathrm{An}_{65}\right)$ and titanomagnetite $\left(X_{\mathrm{mte}}=.48\right)$ from sample 23 (49.4 $\mathrm{wt} \% \mathrm{SiO}_{2}$ on a normalized anhydrous basis) in order to derive a residual liquid similar to sample 303 (55.8 wt $\% \mathrm{SiO}_{2}$ ). The model predicts subtraction of $\sim 8 \%$ olivine, $\sim 19 \%$ augite, $\sim 29 \%$ plagioclase and $\sim 4 \%$ titanomagnetite. The composition of the resultant residual liquid compares well with sample 303, except for the $\mathrm{Fe}_{2} \mathrm{O}_{3}$ and $\mathrm{K}_{2} \mathrm{O}$ components. The relative oxygen fugacity of the resultant liquid, however, is $1.6 \mathrm{log}$ units below the $\mathrm{Ni}-\mathrm{NiO}$ buffer $(\Delta \mathrm{NNO}=-1.6)$.

The second stage involves crystal fraction of the average observed phenocryst compositions: olivine $\left(\mathrm{Fo}_{74}\right)$, augite $\left(\mathrm{En}_{40} / \mathrm{En}_{15}\right)$, plagioclase $\left(\mathrm{An}_{54}\right)$ and titanomagnetite $\left(X_{\mathrm{mte}}=0.56\right)$ from sample 303 (with an initial ferric-ferrous ratio equivalent to $\Delta \mathrm{NNO}=-1.6$ ) in order to derive a residual liquid similar to sample 162 $\left(60.5 \mathrm{wt} \% \mathrm{SiO}_{2}\right)$. This model predicts subtraction of $\sim 6 \%$ olivine, $\sim 19 \%$ plagioclase, and $\sim 2.5 \%$ titanomagnetite and the addition of $\sim 1 \%$ augite. The composition of the residual liquid is similar to sample 162 , except for the $\mathrm{TiO}_{2}, \mathrm{Fe}_{2} \mathrm{O}_{3}$ and $\mathrm{K}_{2} \mathrm{O}$ components. Moreover, the relative oxygen fugacity of the residual liquid is now $\triangle \mathrm{NNO}=-8.8$, which is well below the iron-wüstite buffer. In other words, derivation of the Aurora Crater two-pyroxene andesite (sample 162) by crystal fractionation from a basalt similar to sample 23 , requires that the residual liquid be in equilibrium with metallic iron, which is, of course, unrealistic.

If, alternatively, the second fractionation step is repeated, but this time with an initial ferric-ferrous ratio in sample 303 equivalent to $\Delta \mathrm{NNO}=0$, a similar proportion of subtracted phases is predicted (Table 14), but the drop in $\triangle \mathrm{NNO}$ of the residual liquid is not so severe and is only $-2.0 \log$ units. Nonetheless, this two-stage model requires that some oxidation mechanism be invoked between stages 1 and 2, in order to get the basaltic andesite 
Fig. 9 A series of diagrams illustrating the results of the MELTS calculation for fractional crystallization of sample 23 at $2 \mathrm{kbar}$ and with $1.5 \mathrm{wt} \%$ $\mathrm{H}_{2} \mathrm{O}$. (See text for full description)
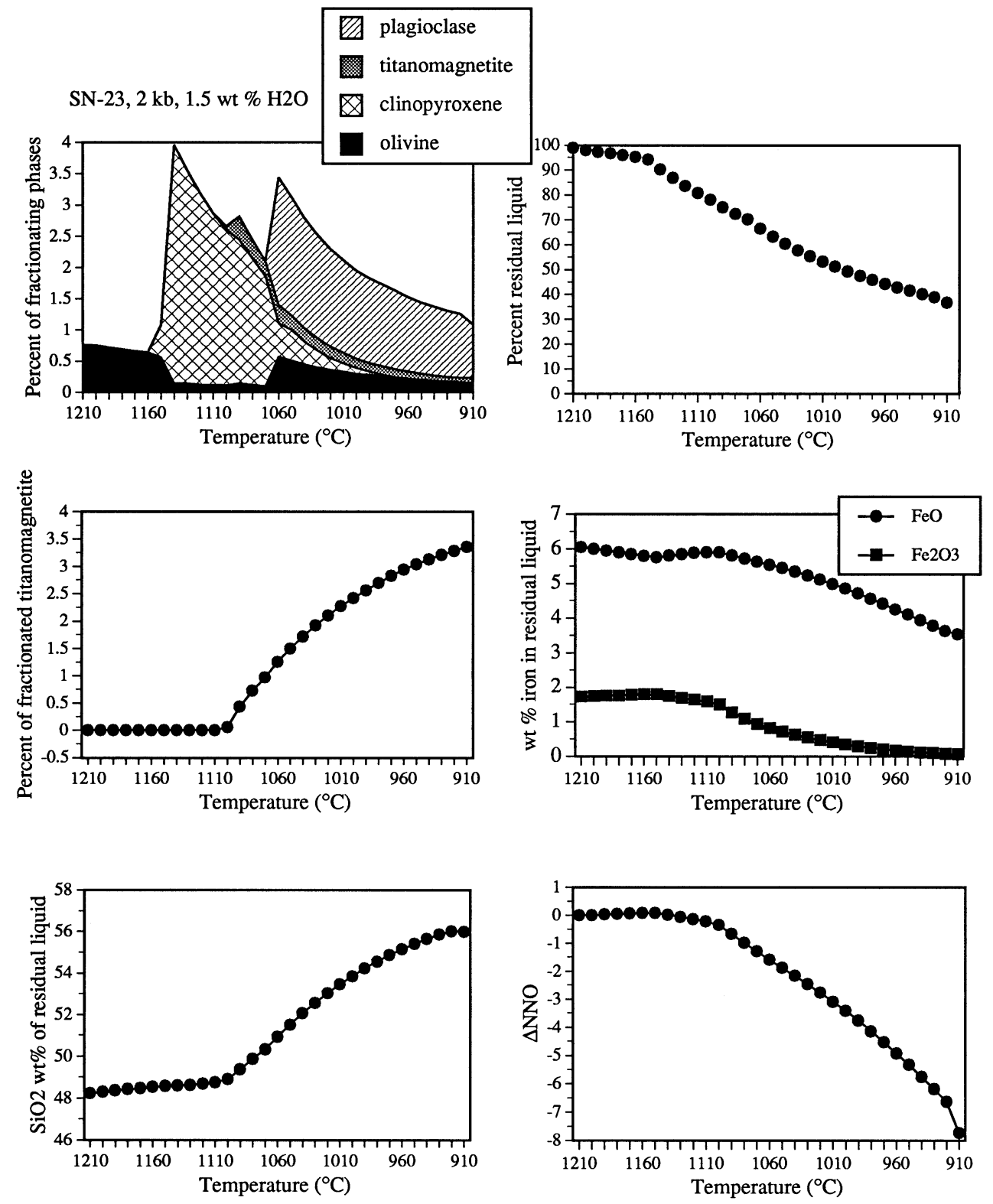

back up to $\triangle \mathrm{NNO}=0$ (an increase of $1.6 \mathrm{log}$ units) after crystal fractionation from a basalt. And then a second oxidation event must take place to get sample 162 back up to $\Delta \mathrm{NNO}=0$ (an increase of $2.0 \log$ units).

The effect of crystal fractionation on the relative oxygen fugacity of residual liquids can also be explored using the MELTS thermodynamic model of Ghiorso and Sack (1995). The advantage of MELTS is that instead of specifying the phase assemblage to be fractionated, as in XLFRAC, the model predicts the composition and proportion of phases that are stable at each increment of cooling. The ferric-ferrous ratio in the intial liquid (sample 23) was chosen to be that calculated in equilibrium with the Ni-NiO buffer (Fig. 7) at the starting temperature and pressure. During fractional crystallization, however, the oxygen fugacity was not imposed, but was allowed to change and be defined by the changing $T$, bulk composition and ferric-ferrous ratio of the residual liq- uid. The results for sample 23 , with an initial water content of $1.5 \mathrm{wt} \%$ and a crystallization pressure of $2 \mathrm{kbar}$ are presented in Fig. 9. (The effect of varying the initial water content and crystallization pressure is discussed below.) In this diagram, the percentage of each fractionating phase at each temperature is presented. At $1210^{\circ} \mathrm{C}$, the liquidus phase is olivine, followed by plagioclase at $1160^{\circ} \mathrm{C}$, titanomagnetite at $1110^{\circ} \mathrm{C}$, and augite at $1070^{\circ} \mathrm{C}$. Approximately $65 \%$ crystallization occurs over the first 300 degrees below the liquidus $\left(1210-910^{\circ} \mathrm{C}\right)$. The concentration of silica in the residual liquid increases slowly from 48 to $49 \mathrm{wt} \%$ over the first 100 degrees of cooling, until titanomagnetite hits the liquidus at $1110^{\circ} \mathrm{C}$. At this point, the $\mathrm{SiO}_{2}$ content increases rapidly from 49 to $56 \mathrm{wt} \%$, whereas both the $\mathrm{FeO}$ and $\mathrm{Fe}_{2} \mathrm{O}_{3}$ components decrease in concentration through removal of the magnetite component in the titanomagnetite phase. Neither the activity of silica nor the oxygen fugac- 
ity in the residual liquid is buffered during fractional crystallization. Therefore, just as the relative activity of silica (corrected for the effect of changes with temperature) in the residual melt changes with the concentration of $\mathrm{SiO}_{2}$, so does the relative oxygen fugacity of the residual liquid drop as its $\mathrm{Fe}^{3+} / \Sigma \mathrm{Fe}$ ratio decreases. In the case presented in Fig. 9, the oxygen fugacity of the residual liquid during fractional crystallization approaches 8 $\log$ units below the Ni-NiO buffer, a result similar to that predicted by the XLFRAC model.

The most important feature of both the XLFRAC and MELTS calculations is that fractional crystallization of only small quantities of titanomagnetite $(\sim 3-4 \%)$ are sufficient to drop the ferric iron concentration in residual liquids to extremely low values. Increasing pressure from 2 to $10 \mathrm{kbar}$ and increasing the initial water content of the parent liquid will not affect this conclusion, since titanomagnetite will remain stable (Grove and Baker 1984; Sisson and Grove 1993). This problem of titanomagnetite fractionation leading to reduced residual liquids was also pointed out by Sisson and Grove (1993) in their discussion of the calc-alkaline trend. The only way to offset this trend is to (i) fractionate ilmenite or hornblende as an alternative to titanomagnetite, or (ii) lose $\mathrm{H}_{2}$ (or $\mathrm{H}_{2} \mathrm{~S}$ ) preferentially through degassing and thereby oxidize the magma; the feasibility of these mechanisms is discussed in the following sections.

\section{Crystal fractionation of ilmenite and/or hornblende}

Fractionation of ilmenite is unlikely during differentiation of the Aurora magmas since it is not found as a phenocryst or in the groundmass in any sample. In basaltic through andesitic liquid compositions, ilmenite is stable only under conditions of high $\mathrm{FeO}$ and $\mathrm{TiO}_{2}$ content (Juster et al. 1989; Synder et al. 1993; Toplis and Carroll 1995). In the Aurora suite, the basalt through andesite liquids are not sufficiently enriched in titanium or ferrous iron to stabilize ilmenite as a phenocryst or in the groundmass. Therefore, ilmenite cannot be invoked as a fractionating phase in a basalt or basaltic andesite parent.

Fractionation of hornblende as an alternative to titanomagnetite has frequently been invoked to generate the early silica enrichment and iron depletion that characterizes the calc-alkaline differentation trend (e.g., Lewis 1973; Cawthorn and O'Hara 1976; Green 1982; Foden 1983). There are two difficulties with this hypothesis. Firstly, the $\mathrm{Fe}^{3+} / \Sigma \mathrm{Fe}$ ratios in the carefully separated phenocryst cores (Table 9) are approximately the same as those in coexisting titanomagnetite. Thus fractionation of hornblende will lead to the same ferric iron depletion in the residual liquid as found for titanomagnetite. Secondly, numerous experiments have demonstrated that hornblende is not stable at temperatures greater than $\sim 1065^{\circ} \mathrm{C}$ (e.g., Eggler 1972; Holloway and Burnham 1972; Allen and Boettcher 1978; Helz 1982; Foden and Green 1992). Two-pyroxene thermometry indicates that the Aurora Crater two-pyroxene andesite (sample 162) erupted at a temperature of $\sim 1069 \pm 5^{\circ} \mathrm{C}$. Any magma that was parental to sample 162 through a crystal fractionation process must have been hotter, and therefore could not have had hornblende on its liquidus.

The effect of degassing on the oxygen fugacity of residual liquids

A commonly invoked mechanism for the oxidation of magmatic liquids is through preferential loss of either the $\mathrm{H}_{2}, \mathrm{H}_{2} \mathrm{~S}$ or $\mathrm{CO}$ component during degassing (Sato 1978; Mathez 1984; Sisson and Grove 1993). However, this postulate is in direct contrast to the conclusions drawn by Gerlach (1993) in a detailed study of the degassing of magma at Kilauea Volcano, Hawaii. Gerlach (1993) showed that volcanic gases collected along the east rift zone of Kilauea, over a temperature interval of 1185 to $935^{\circ} \mathrm{C}$, have equilibrium species concentrations that "...show unequivocally that coexisting lavas buffered the gas oxygen fugacities during cooling." The gases are divided into a Type I (released in sustained summit lava lake eruptions after relatively short periods of crustal storage) and a Type II (derived from evolved basalts that experienced prolonged storage in the crust). The $\log f_{\mathrm{O}_{2}}$-temperature path of both Type I and II gases parallels the Ni-NiO and QFM buffer curves and has a constant value of $\triangle \mathrm{NNO}=-0.51 \pm 0.04$. This value is consistent with those calculated for Kilauean lavas based on Fe-Ti oxides and bulk ferric-ferrous ratios in glassy lavas (Gerlach 1993). The evidence from Kilauea demonstrates, therefore, that the oxygen fugacity of the exsolved gases is controlled by that of the host magma, and that the $\triangle \mathrm{NNO}$ value of the host magma does not change even after substantial degassing, cooling and closed system crystallization.

\section{Equilibrium crystallization}

An alternative to fractional crystallization is equilibrium crystallization, where phenocrysts remain in contact with the residual liquid. In this case, the relative oxygen fugacity (and ferric-ferrous ratio) of the residual liquid will not deviate significantly from that of the initial melt, because of the buffering capacity of the crystallizing assemblage: olivine, pyroxene and spinel. Although these phases are solid solutions, and their buffering capacity is multivariant, the $f_{\mathrm{O}_{2}}$ of the residual liquid is not expected to change substantially from that of the parent liquid. In fact, the evidence from Kilauea (Gerlach 1993) is that closed system crystallization does not change the $f_{\mathrm{O}_{2}}$ of the bulk magma within $\pm 0.5 \log$ units. However, progressive tapping of magma chambers during equilibrium crystallization, where crystals are required to remain in contact with the liquid, is inconsistent with the eruption of several, nearly aphyric basaltic andesite and andesite lavas (e.g., samples 303, 311, 160, 162, 329, 
319; Table 2) in the Aurora field. A more realistic scenario involves the accumulation of mantle-derived magmas at the base of the crust where they solidify under conditions closer to equilibrium than fractional crystallization, and are subsequently remelted during intrusion of fresh batches of basalt.

This is not to say that fractional crystallization of basaltic magma never occurs; layered plutonic cumulate rocks (ultramafic and gabbroic) are frequently associated with noncumulate gabbros, diorites and granodiorites in zoned intrusive complexes in arc environments (e.g., Snoke et al. 1981). However, the cumulate rocks are frequently subordinate to the noncumulate rocks, and the layered, cumulate texture is generally absent in the more evolved plutonic bodies (diorite-granodiorite-granite). Thus, fractionation may be an appropriate model for early crystallization of a basalt, in contrast to equilibrium crystallization by the time titanomagnetite joins the liquidus.

\section{Partial melting of young, mafic lower crust}

The lower crust is an obvious location for the aggregation of mantle-derived melts, where they become neutrally buoyant. There is a growing body of geophysical evidence for the accumulation of basaltic magma in the lower crust, including the presence of well-defined subhorizontal reflections (Mereu et al. 1989). It is conceivable that repeated intrusion of basalt into the lower crust may lead to partial melting of previous underplates. Such a scenario has been proposed for calc-alkaline volcanic and plutonic suites by several others (e.g., Kay and Kay 1991; Atherton and Petford 1993), and is indistinguishable from the MASH (melting, assimilation, storage, assimilation) hypothesis of Hildreth and Moorbath (1988) that invoked partial melting of recently crystallized mafic magma lodged in the lower crust. Petrologic evidence for such a process is found in numerous granulite xenoliths entrained in a variety of lavas (e.g., Rogers and Hawkesworth 1982; Griffin and O'Reilly 1986; Wilshire 1990; Hickey-Vargas et al. 1995).

Based on a series of phase equilibrium experiments, Rushmer (1991) presented a model for the volume of melt produced during fluid-absent melting of a mafic source rock (i.e., amphibolitized basalt). At $10 \mathrm{kbar}$, between 800 and $1000^{\circ} \mathrm{C}$, the percentage of melt produced can vary between $10-40 \%$ if the source rock contains $1 \mathrm{wt} \%$ total water. Both the volume of melt produced, and hence its composition, can vary significantly during dehydration melting as a function of reasonable variations in either temperature or the water content of the source rock. It is plausible, therefore, that a continuum of melt compositions could be generated during partial melting of previously crystallized, variably amphibolitized, basaltic magma.

A model that favors differentiation by repeated partial melting of recently crystallized basaltic magmas in the lower crust, rather than by crystal fractionation in a large, stable magma chamber in the upper crust, has implications for the thermal regime and magma supply rate expected during evolution of the Aurora calc-alkaline trend. As discussed above, the average eruption rate during the approximately 3.6 million year lifetime of the Aurora volcanic field is roughly equivalent to the "background trickle" at the Mt Adams volcanic field (Hildreth and Lanphere 1994) and is an order of magnitude less than that of the slow-spreading Mid-Atlantic ridge (BVTP 1981). Hildreth and Lanphere (1994) used the low average eruption rate at Mt Adams to argue against the development of an upper-crustal magma chamber beneath that stratovolcano. Their conclusion, combined with the realization that largely-molten reservoirs are equally ephemeral at slow spreading ridges (Sinton and Detrick 1992), supports the idea that maintenance of a stable magma chamber beneath the Aurora volcanic field was unlikely.

A model for calc-alkaline differentiation from basalt to dacite through partial melting of young, mafic lower crust is consistent with the conclusions drawn from several detailed field and geochemical studies of calc-alkaline, zoned and layered plutons (e.g., Gromet and Silver 1987; Tepper et al. 1993; Coleman et al. 1992, 1995). For example, the Lamark granodiorite and Goodale granite in the east-central Sierra Nevada are underlain by large mafic sill complexes (Aberdeen and Onion Valley) described by Frost and Mahood (1987), Sisson (1991) and Coleman et al. (1992, 1995). On the basis of isotopic data from the Lamarck intrusion, Coleman et al. (1992) argued that the granodiorite was formed through partial melting of hornblende gabbros and diorites similar in composition to those exposed in the mafic sill complexes. In a detailed field and geochemical study of the Chilliwack batholith in the Cascades, Tepper et al. (1993) concluded that partial melting of previously crystallized high-Al basalts (containing varying amounts of water) could produce the spectrum of compositions found in calc-alkaline plutons. Similarly, Gromet and Silver (1987) argued that the REE (rare-earth element) trends across the Peninsular Ranges batholith cannot be explained by fractional crystallization, assimilation, combined crystallization-assimilation, or mixing processes; instead, they concluded that the geochemical and petrologic features of the tonalites and granodiorites of the Peninsular Ranges require an origin through partial melting of mafic source rocks. The evidence of partial melting in calc-alkaline plutonic environments is entirely consistent with the low eruption rate at the Aurora volcanic field, with the oxygen fugacities and bulk compositions of the Aurora lavas, and with the absence of any relationship between the age of an Aurora lava and its composition.

Acknowledgements The authors acknowledge partial support from NSF grants EAR-9405768 (to RAL) and EAR-9415844 (to ISEC). This study would never have been initiated were it not for a field trip taken with Professor Charles Gilbert in the summer before he died, while the authors were teaching field camp. His vast knowledge and love of the area, now commemorated in the 
Gilbert Peak of Long Valley where he discovered the Bishop Tuff almost sixty years before, was an inspiration to the authors and the undergraduates of a summer field camp. Assistance in the field from several undergraduates, as well as Dawnika Blatter and Gordon Moore, is greatly appreciated. Constructive comments from Jake Lowenstern, Tim Grove, and an anonymous reviewer significantly improved the manuscript.

\section{References}

Allen JC, Boettcher AL (1978) Amphiboles in andesite and basalt: II. Stability as a function of P-T- $f_{\mathrm{O}_{2}}$. Am Mineral 63:10741087

Atherton MP, Petford N (1993) Generation of sodium-rich magmas from newly underplated basaltic crust. Nature 362:144146

Atwater T (1970) Implications of plate tectonics for the Cenozoic tectonic evolution of western North America. Geol Soc Am Bull 81:3513-3536

Bacon CR, Metz J (1984) Magmatic inclusions in rhyolites, contaminated basalts, and compositional zonation beneath the $\mathrm{Co}-$ so volcanic field, California. Contrib Mineral Petrol 85:346365

Baker DR, Eggler DH (1987) Compositions of anhydrous and hydrous melts coexisting with plagioclase, augite, and olivine or low-Ca pyroxene from 1 atm to 8 kbar:application to the Aleutian volcanic center of Atka. Am Mineral 72:12-28

BVTP (1981) Basaltic volcanism on the terrestrial planets (Lunar and Planetary Institute). Pergamon, New York

Carmichael ISE (1967) The iron-titanium oxides of salic volcanic rocks and their associated ferromagnesian silicates. Contrib Mineral Petrol 14:36-64

Cawthorn GR, O'Hara MJ (1976) Amphibole fractionation in calcalkaline magma genesis. Am J Sci 276:309-329

Chesterman CW (1968) Volcanic history of the Bodie Hills. Geol Soc Am Mem 116:45-68

Coleman DS, Frost TP, Glazner AF (1992) Evidence from the Lamarck Granodiorite for rapid Late Cretaceous crust formation in California. Science 258: 1924-1926

Coleman DS, Glazner AF, Miller JS, Bradford KJ, Frost TP, Joyce JL, Bachl CA (1995) Exposure of a Late Cretaceous layered mafic-felsic magma system in the central Sierra $\mathrm{Ne}$ vada batholith, California. Contrib Mineral Petrol 120:129136

Cosca MA, Essene EJ, Bowman JR (1991) Complete chemical analyses of metamorphic hornblendes:implications for normalizations, calculated $\mathrm{H}_{2} \mathrm{O}$ activities and thermobarometry. Contrib Mineral Petrol 108:472-484

Eggler DH (1972) Amphibole stability in $\mathrm{H}_{2} \mathrm{O}$-undersaturated calc-alkaline melts. Earth Planet Sci Lett 15:28-34

Feldstein SN, Lange RA (1996) Pliocene potassic magmas from the Kings River region, Sierra Nevada, California: evidence for melting a veined subarc mantle during extension. J Petrol (in review)

Feldstein SN, Lange RA, Vennemann T, O'Neil JR (1996) Ferricferrous ratios, $\mathrm{H}_{2} \mathrm{O}$ contents and $\mathrm{D} / \mathrm{H}$ ratios of phlogopite and biotite from lavas of different tectonic regimes. Contrib Mineral Petrol (in press)

Fitton JG, James D, Leeman WP (1991) Basic magmatism associated with Late Cenozoic extension in the western United States:compositional variations in space and time. J Geophys Res 96: 13693-13711

Foden JD (1983) The petrology of the calc-alkaline lavas of Rindjani volcano, East Sunda Arc: a model for island arc petrogenesis. J Petrol 24:98-130

Foden JD, Green DH (1992) Possible role of amphibole in the origin of andesite: some experimental and natural evidence. Contrib Mineral Petrol 109:479-493

Frost TP, Mahood GA (1987) Field, chemical, and physical constraints on mafic-felsic magma interaction in the Lamarck
Granodiorite, Sierra Nevada, California. Geol Soc Am Bull 99: 272-291

Gerlach TM (1993) Oxygen buffering of Kilauea volcanic gases and the oxygen fugacity of Kilauea basalt. Geochim Cosmochim Acta 57: 795-814

Ghiorso MS, Sack RO (1995) Chemical mass transfer in magmatic processes IV. A revised and internally consistent thermodynamic model for the interpolation and extrapolation of liquidsolid equilibria in magmatic systems at elevated temperatures and pressures. Contrib Mineral Petrol 119: 197-212

Gilbert CM, Christensen MN, Al-Rawi Y, Lajoie KL (1968) Structural and volcanic history of Mono Basin, California-Nevada. Geol Soc Am Mem 116:275-329

Gill JB (1981) Orogenic andesites and plate tectonics. Springer, Berlin

Green TH (1982) Anatexis of mafic crust and high pressure crystallization of andesites. In: Thorpe RS (ed) Andesite: orogenic andesites and related rocks. Wiley, Chichester, pp 465-487

Griffin WL, O'Reilly SY (1986) The lower crust in eastern Aus tralia: xenolith evidence. In: Dawson JB et al. (eds) The nature of the continental crust. Geol Soc London Spec Pub 24:363374

Gromet LP, Silver LT (1987) REE variations across the Peninsular Ranges batholith:implications for batholithic petrogenesis and crustal growth in magmatic arcs. J Petrol 28:75-125

Grove TL, Baker MB (1984) Phase equilibrium controls on the tholeiitic versus calc-alkaline differentiation trends. J Geophys Res 89: 3253-3274

Grove TL, Juster TC (1989) Experimental investigations of low-Ca pyroxene stability and olivine-pyroxene-liquid equilibria at 1 atm in natural basaltic and andesitic liquids. Contrib Mineral Petrol 103: 287-305

Grove TL, Kinzler RJ (1986) Petrogenesis of andesites. Ann Rev Earth Planet Sci Lett 14:417-454

Grove TL, Kinzler RJ, Baker MJ, Donnely-Nolan JM, Lesher CE (1988) Assimilation of granite by basaltic magma at Burnt Lava flow, Medicine Lake volcano, northern California:decoupling of heat and mass transfer. Contrib Mineral Petrol 99:320-343

Hasenaka T (1994) Size, distribution, and magma output rate for shield volcanoes of the Michoacán-Guanajuato volcanic field, Central Mexico. J Volcanol Geotherm Res 63: 13-31

Helz RT (1982) Phase relationships and compositions of amphiboles produced in studies of the melting behavior or rocks. In: Veblen DR, Ribbe PH (eds) Amphiboles: petrology and experimental phase relations (Reviews in Mineralogy, vol. 9B) Mineralogical Society of America, Washington DC, pp 279346

Hickey-Vargas R, Abdollahi MJ, Parada MA, López-Escobar L, Frey FA (1995) Crustal xenoliths from Calbuco volcano, Andean southern volcanic zone: implications for crustal composition and magma-crust interaction. Contrib Mineral Petrol 119:331-344

Hildreth W, Lanphere MA (1994) Potassium-argon geochronology of a basalt-andesite-dacite arc system: the Mount Adams volcanic field, Cascade Range of southern Washington. Geol Soc Am Bull 106: 1413-1429

Hildreth W, Moorbath S (1988) Crustal contributions to arc magmatism in the Andes of central Chile. Contrib Mineral Petrol 98: 455-489

Holloway JR, Burnham CW (1972) Melting relations of basalt with equilibrium water pressure less than total pressure. J Petrol 13:1-29

Juster TC, Grove TL, Perfit MR (1989) Experimental constraints on the generation of Fe-Ti basalts, andesites, and rhyodacites at the Galapagos spreading center, $85^{\circ} \mathrm{W}$ and $95 \mathrm{~W}$. J Geophys Res 94: 9251-9274

Kay RW, Kay SM (1991) Creation and destruction of lower continental crust. Geol Rundsch 80: 259-278

Kelleher PC, Cameron KL (1990) The geochemistry of the Mono Craters-Mono Lake Islands volcanic complex, eastern California. J Geophys Res 95: 17643-17659 
Kempton PD, Fitton JG, Hawkesworth CJ, Ormerod DS (1991) Isotopic and trace element constraints on the composition and evolution of the lithosphere beneath the southwestern United States. J Geophys Res 96:13713-13735

Kistler RW, Peterman ZE (1978) Reconstruction of crustal bolcks of California on the basis of initial strontium isotopic compositions of Mesozoic granitic rocks. US Geol Surv Prof Pap 1071

Kress VC, Carmichael ISE (1991) The iron redox state in silicate liquids:the effect of pressure, temperature, oxygen fugacity and composition, with application to the genesis of basic magma. Contrib Mineral Petrol 108: 82-92

Lange RA, Carmichael ISE, Renne P (1993) Potassic volcanism near Mono Basin, California: evidence for high water and oxygen fugacities inherited from subduction. Geology 21:949952

Leeman WP (1970) The isotopic composition of strontium in lateCenozoic basalts from the Basin-Range province, western United States. Geochim Cosmochim Acta 34: 857-872

Lewis JF (1973) Petrology of ejected plutonic blocs of the Soufriere volcano, St. Vincent, West Indies. J Petrol 14: 81112

Lindsley DH, Frost BR (1992) Equilibria among Fe-Ti oxides, pyroxenes, olivine and quartz: part 1. Theory. Am Mineral 77: $987-1003$

Luhr JF (1990) Experimental phase relations of water- and sulfursaturated arc magmas and the 1982 eruptions of El Chichón volcano. J Petrol 31:1071-1114

Mathez EA (1984) Influence of degassing on oxidation states of basaltic magmas. Nature 310:371-375

Mereu RF, Mueller S, Fountain DM (1989) Properties and processes of earth's lower crust. Geophys Monogr Am Geophys Union 51

Michael PJ (1991) Intrusion of basaltic magma into a crystallizing granitic magma chamber: the Cordillera del Paine pluton in southern Chile. Contrib Mineral Petrol 108:396-418

Miyashiro A (1974) Volcanic rock series in island arcs and active continental margins. Am J Sci 274:321-355

Moore G (1995) Experimental and field studies of hydrous magmas. PhD dissertation, University of California, Berkeley

Moore G, Righter K, Carmichael ISE (1995) The effect of dissolved water on the oxidation state of iron in natural silicate liquids. Contrib Mineral Petrol 120: 170-179

Moore G, Lange RA, Carmichael ISE (1996) Olivine-liquid equilibria and oxygen fugacity in hydrous, basic magmas: examples from Oregon, eastern California and western Mexico. Am Mineral (submitted)

Moore JG, Dodge FCW (1980) Late Cenozoic volcanic rocks of the southern Sierra Nevada, California: I. Geology and petrology. Geol Soc Am Bull 91: 1995-2038

Nielsen D, Mahood G, Halliday A, Essene E (1991) Petrogenesis of precaldera mafic and intermediate lavas, Long Valley, CA. Geol Soc Am Abstr 23:A83

Ormerod DS, Hawkesworth CJ, Rogers NW, Leeman WP, Menzies MA (1988) Tectonic and magmatic transitions in the western Great Basin, USA. Nature 333:349-353

Osborn EF (1959) Role of oxygen pressure in the crystallization and differentiation of basaltic magma. Am J Sci 257: 609-647

Pallister JS, Hoblitt RP, Reyes AG (1992) A basalt trigger for the 1991 eruptions of Pinatubo volcano? Nature 356: 426-428

Panjasawatwong Y, Danyushevsky LV, Crawford AJ, Harris KL (1995) An experimental study of the effects of melt composition on plagioclase-melt equilibria at 5 and 10 kbar: implications for the origin of magmatic high-An plagioclase. Contrib Mineral Petrol 118:420-432

Righter K, Carmichael ISE (1996) Phase equilibria of phlogopite lamprophyres from western Mexico: biotite-liquid equilibria and $P-T$ estimates for biotite-bearing igneous rocks. Contrib Mineral Petrol 123:1-21

Rogers NW, Hawkesworth CJ (1982) Proterozoic age and cumulate origin for granulite xenoltihs, Lesotho. Nature 299:409413
Rushmer T (1991) Partial melting of two amphibolites:contrasting experimental results under fluid-absent conditions. Contrib Mineral Petrol 107: 41-59

Rutherford MJ, Devine JD (1988) The May 181980 eruption of Mount St Helens 3. Stability and chemistry of amphibole in the magma chamber. J Geophys Res 93:11949-11959

Sato M (1978) Oxygen fugacity of basaltic magmas and the role of gas-forming elements. Geophys Res Lett 5:447-449

Silberman ML, Chesterman CW (1972) K-Ar age of volcanism and mineralization, Bodie mining district and Bodie Hills volcanic field, Mono county, California. Isochron/West 3:13-22

Silberman ML, McKee EH (1972) A summary of radiometric age determinations on Tertiary volcanic rocks from Nevada and eastern California: part II, western Nevada. Isochron/West $4: 7-28$

Sinton JM, Detrick RS (1992) Mid-ocean ridge magma chambers. J Geophys Res 97: 197-216

Sisson TW (1991) Field, geochemical, and experimental studies of aluminous arc magmas. PhD dissertation, Massachusetts Institute of Technology

Sisson TW, Grove TL (1993) Experimental investigations of the role of $\mathrm{H}_{2} \mathrm{O}$ in calc-alkaline differentiation and subduction zone magmatism. Contrib Mineral Petrol 113:143-166

Snoke AW, Quick JE, Bowman HR (1981) Bear Mountain igneous complex, Klamath Mountains, an ultrabasic to silicic calc-alkaline suite. J Petrol 22:501-552

Snyder D, Tait S (1996) Magma mixing by convective entrainment. Nature 379:529-531

Snyder D, Carmichael ISE, Wiebe RA (1993) Experimental study of liquid evolution in an Fe-rich, layered mafic intrusion:constraints of Fe-Ti oxide precipitation on the $\mathrm{T}-f_{\mathrm{O}_{2}}$ and T- $\varrho$ paths of tholeiitic magmas. Contrib Mineral Petro 113:73-86

Speer JA (1984) Crystal chemistry of the true micas. In: Bailey SW (ed) Micas (Reviews in Mineralogy vol 13); Mineralogical Society of America, Washington DC, pp 299-356

Stormer JC, Nicholls J (1978) XLFRAC: a program for interactive testing of magmatic differentiation models. Computers and Geoscience 4: 143-159

Tepper JH, Nelson BK, Bergantz GW, Irving AJ (1993) Petrology of the Chilliwack Batholith, North Cascades, Washington: generation of calc-alkaline granitoids by melting of mafic lower crust with variable water fugacity. Contrib Mineral Petro 113:333-351

Toplis MJ, Carroll MR (1995) An experimental study of the influence of oxygen fugacity on Fe-Ti oxide stability, phase relations, and mineral-melt equilibria in ferro-basaltic systems. J Petrol 36: 1137-1170

Tsuchiyama A (1985) Dissolution kinetics of plagioclase in the melt of the system diopside-albite-anorthite, and origin of dusty plagioclase in andesites. Contrib Mineral Petrol 89: 1-16

Van Kooten GK (1980) Mineralogy, petrology and geochemistry of an ultrapotassic suite, central Sierra Nevada, California, U.S.A. J Petrol 21:651-684

Van Kooten GK (1981) Pb and Sr systematics of ultrapotassic and basaltic rocks from the central Sierra Nevada, California. Contrib Mineral Petrol 76: 651-684

Van Kooten GK, Leeman WP, Menzies MA (1985) Lithospheric peridotites and pyroxenites in alkaline basaltic rock from the Sierra Nevada, California, U.S.A. EOS, Trans Am Geophys Union 66: 414

Vogel TA, Woodburne TB, Eichelberger JG, Layer PW (1994) Chemical evolution and periodic eruption of mafic lava flows in the west moat of Long Valley caldera, California. J Geophys Res 99: 19829-19842

Wiebe RA (1993) The Pleasant Bay layered gabbro-diorite, coastal Maine: ponding and crystallization of basaltic injections into a silicic magma chamber. J Petrol 34:461- 489

Wilshire HG (1990) Lithology and evolution of the crust-mantle boundary region in the southwestern Basin and Range province. J Geophys Res 95:649-665 